ESAIM: M2AN 50 (2016) 905-920

DOI: $10.1051 / \mathrm{m} 2 \mathrm{an} / 2014064$
ESAIM: Mathematical Modelling and Numerical Analysis

www.esaim-m2an.org

\title{
THE ELECTROMAGNETIC SCATTERING PROBLEM WITH GENERALIZED IMPEDANCE BOUNDARY CONDITIONS
}

\author{
Nicolas Chaulet ${ }^{1}$
}

\begin{abstract}
In this paper we consider the electromagnetic scattering problem by an obstacle characterised by a Generalized Impedance Boundary Condition in the harmonic regime. These boundary conditions are well known to provide accurate models for thin layers or imperfectly conducting bodies. We give two different formulations of the scattering problem and we provide some general assumptions on the boundary condition under which the scattering problem has at most one solution. We also prove that it is well-posed for three different boundary conditions which involve second order surface differential operators under weak sign assumptions on the coefficients defining the surface operators.
\end{abstract}

Mathematics Subject Classification. 35P25, 35G05, 35Q61, 78A45.

Received November 18, 2013. Revised November 24, 2014.

Published online May 23, 2016.

\section{INTRODUCTION}

Driven by recent advances in the study of inverse acoustic scattering problems in the presence of so-called generalized impedance boundary conditions (see $[2-4,6]$ ) we study in this paper well-posedness of the forward electromagnetic scattering problem in the harmonic regime in the case where the scatterer is characterised by a boundary condition of the form

$$
\boldsymbol{\nu} \times \mathbf{E}+\mathcal{Z} \mathbf{H}_{T}=\mathbf{f} \text { on } \Gamma
$$

where $\Gamma$ is the boundary of the scatterer, $\boldsymbol{\nu}$ is the outward unit normal vector to $\Gamma, \mathbf{E}$ is the electric field, $\mathbf{H}_{T}$ stands for the tangential component of the magnetic field $\mathbf{H}, \mathcal{Z}$ is a surface differential operator and $\mathbf{f}$ is a source term. This kind of boundary conditions, often referred to as Generalized Impedance Boundary Condition, are known to provide accurate models for all sort of small scale structures. Moreover, in some specific configurations, such as the scattering by a perfect conductor covered by a thin layer of dielectric or of ferromagnetic material (see $[1,11,13]$ ), or the scattering by an imperfectly conducting body (see [14]), asymptotic analysis techniques provide an expression for $\mathcal{Z}$ in terms of surface differentials operators as well as approximation properties. For a similar treatment of transmission problems see $[7,12]$ for example.

In this paper we establish sufficient conditions on the operator $\mathcal{Z}$ under which the scattering problem is well posed. We introduce two different ways of writing the problem: the first one (which we will call the volume approach) consists in considering the scattering problem as a volume problem and in studying the associated

Keywords and phrases. Maxwell's equations, generalized impedance boundary conditions, electromagnetic scattering, Helmholtz' decomposition.

1 Department of Mathematics, University College London, Gower street, London, WC1E 6BT, UK. nicolas.chaulet@gmail.com 
variational formulation. This path is rather standard and follows the lines of ([15], Chap. 10). We state a general existence and uniqueness result for the scattering problem which uses the volume formulation in Theorem 3.6. Nevertheless, with these standard approach one needs to assume some compatibility between the signs of the surface operator $\mathcal{Z}$ and the sign of the volume contribution to the variational formulation to ensure reasonable coercivity properties. Actually, at least for the acoustic scattering problem problem (see $[6,16]$ ), it seems that such restrictive conditions are not needed. To clarify this point, we consider a different formulation for the scattering problem which consists in writing the problem as a single operator equation posed on the boundary of the scatterer. We will call this approach the surface formulation. We indeed show that the scattering problem is equivalent to finding the tangential component of the electromagnetic field $\mathbf{H}$ that solves

$$
\left(\boldsymbol{S}_{\Gamma}+\mathcal{Z}\right) \mathbf{H}_{T}=\mathbf{f} \quad \text { on } \Gamma
$$

where $\boldsymbol{S}_{\Gamma}$ is the so-called Magnetic-to-Electric Calderón operator (see [15], Chap. 9 for example). In the scalar case, it is sufficient to assume that $\mathcal{Z}$ is a pseudo-differential operator of order greater or smaller than 1 to obtain existence and uniqueness of the solution to the scattering problem.

Even though we establish a general existence and uniqueness result for this formulation in Theorem 3.5, the situation is more challenging than in the scalar case mainly because the principal part of $\mathcal{Z}$ may have a kernel of infinite dimension. To tackle this difficulty, we will introduce a tailored Helmholtz' decomposition on the boundary of the scatterer. This allows for example to treat the case of an operator $\mathcal{Z}$ corresponding to the first order impedance boundary condition for thin coatings which is given by

$$
\mathcal{Z}=\frac{i \delta}{\kappa \epsilon} \operatorname{curl}_{\Gamma} \operatorname{curl}_{\Gamma}-i \kappa \mu \delta
$$

where $\operatorname{curl}_{\Gamma}$ and $\operatorname{curl}_{\Gamma}$ stand for the surface vectorial and scalar rotational operators, $\epsilon$ and $\mu$ are the dielectric constants of the coating and $\delta$ is the thickness of the layer. For this operator, the surface approach gives wellposedness regardless the sign of $\epsilon$ (which can be negative for metals) whereas the volume approach seems to be limited to positive $\epsilon$.

In the next section we introduce notations and recall some important concepts for the study of boundary value problems for Maxwell's equations. In the third section, we introduce the volume and surface equations and we give general results about existence and uniqueness. Finally, the fourth section is dedicated to the study of well-posedness for three different surface operators of order 2, each of them requiring the use of different techniques.

\section{Problem Setting and VARiational spaces}

Let $\Omega$ be a simply connected open bounded domain of $\mathbb{R}^{3}$ with $C^{1,1}$ boundary $\Gamma$ and let $\Omega_{\text {ext }}:=\mathbb{R}^{3} \backslash \bar{\Omega}$ be its complementary. We consider the following exterior boundary value problem for the electromagnetic field $(\mathbf{E}, \mathbf{H})$ :

$$
\left\{\begin{array}{l}
\operatorname{curl} \mathbf{H}+i \kappa \epsilon \mathbf{E}=0 \quad \text { in } \Omega_{\mathrm{ext}} \\
\operatorname{curl} \mathbf{E}-i \kappa \mu \mathbf{H}=0 \quad \text { in } \Omega_{\mathrm{ext}} \\
\boldsymbol{\nu} \times \mathbf{E}+\mathcal{Z} \mathbf{H}_{T}=\mathbf{f} \quad \text { on } \Gamma
\end{array}\right.
$$

where $\boldsymbol{\nu} \in\left(C^{0,1}(\Gamma)\right)^{3}$ is the inward unit normal to $\Omega, \mathbf{H}_{T}:=(\boldsymbol{\nu} \times \mathbf{H}) \times \boldsymbol{\nu}, \mathcal{Z}$ is a surface differential operator (see Def. 3.1) and $\mathbf{f}$ is some function defined on $\Gamma$. We also denote by $\kappa>0$ the wavenumber and by $\epsilon$ and $\mu$ the relative permittivity and permeability respectively. Following [8], we will assume that $\epsilon$ and $\mu$ are complex valued function of $C^{1}\left(\mathbb{R}^{3} \backslash \Omega\right)$ with nonnegative imaginary part and positive real part. We also assume that $\mu(x)=\epsilon(x)=1$ for all $x$ outside a fixed ball of radius $R_{0}$. When considering the scattering of an incident wave $\left(\mathbf{E}^{i}, \mathbf{H}^{i}\right)$ that solves

$$
\operatorname{curl} \mathbf{H}^{i}+i \kappa \epsilon \mathbf{E}^{i}=0 \text { and } \operatorname{curl} \mathbf{E}^{i}-i \kappa \mu \mathbf{H}^{i}=0 \text { in } \mathbb{R}^{3},
$$


the right hand side $\mathbf{f}$ is given by

$$
\mathbf{f}:=-\left(\boldsymbol{\nu} \times \mathbf{E}^{i}+\mathcal{Z} \mathbf{H}_{T}^{i}\right) .
$$

We complement equations (2.1) with the so-called Silver-Müller radiation condition

$$
\lim _{R \rightarrow \infty} \int_{\partial B_{R}}|\mathbf{H} \times \hat{x}-(\hat{x} \times \mathbf{E}) \times \hat{x}|^{2} \mathrm{~d} s=0
$$

where $B_{R}$ is a ball of radius $R$ and $\hat{x}:=x /|x|$.

To study equations (2.1) and (2.2) we introduce some classical energy spaces and to define specific surface differential operators. We recall hereafter some classical results from ([5], Chap. 2) for the convenience of the reader. Let $\mathcal{O}$ be a generic bounded simply connected open set of $\mathbb{R}^{3}$ with $C^{1,1}$ boundary $\partial \mathcal{O}$ and with outer unit normal $\boldsymbol{\nu}$. Let us first introduce the usual energy space $\boldsymbol{H}(\mathbf{c u r l}, \mathcal{O})$ of $\left(L^{2}(\mathcal{O})\right)^{3}$ distributions with curl in $\left(L^{2}(\mathcal{O})\right)^{3}$ as well as the space of $L^{2}$ tangential vector fields on $\partial \mathcal{O}$ :

$$
\boldsymbol{L}_{t}^{2}(\partial \mathcal{O}):=\left\{\mathbf{v} \in\left(L^{2}(\partial \mathcal{O})\right)^{3} \mid \mathbf{v} \cdot \boldsymbol{\nu}=0\right\} .
$$

For $s \in[-1,1]$ we denote $\boldsymbol{H}_{t}^{s}(\partial \mathcal{O})$ the closure of $\left\{\mathbf{v} \in\left(C^{\infty}(\partial \mathcal{O})\right)^{3} \mid \mathbf{v} \cdot \boldsymbol{\nu}=0\right\}$ in $\left(H^{s}(\partial \mathcal{O})\right)^{3}$. The tangential trace operators are given for $\mathbf{v} \in\left(H^{1}(\mathcal{O})\right)^{3}$ by

$$
\gamma_{t} \mathbf{v}:=\boldsymbol{\nu} \times\left.\mathbf{v}\right|_{\partial \mathcal{O}}, \quad \mathbf{v}_{T}=\gamma_{T}(\mathbf{v}):=\left(\boldsymbol{\nu} \times\left.\mathbf{v}\right|_{\partial \mathcal{O}}\right) \times \boldsymbol{\nu} .
$$

These two operators are bounded and linear from $\left(H^{1}(\mathcal{O})\right)^{3}$ into $\boldsymbol{L}_{t}^{2}(\partial \mathcal{O})$. Let us now introduce the surface differential operators $\nabla_{\partial \mathcal{O}}: H^{1}(\partial \mathcal{O}) \rightarrow \boldsymbol{L}_{t}^{2}(\partial \mathcal{O})$ and $\operatorname{curl}_{\partial \mathcal{O}}: H^{1}(\partial \mathcal{O}) \rightarrow \boldsymbol{L}_{t}^{2}(\partial \mathcal{O})$ that are given for $u \in$ $H^{1}(\partial \mathcal{O})$ by

$$
\nabla_{\partial \mathcal{O}} u:=\gamma_{T}(\nabla \widetilde{u}) \text { and } \operatorname{curl}_{\partial \mathcal{O}} u:=-\boldsymbol{\nu} \times \nabla_{\partial \mathcal{O}} u,
$$

where $\widetilde{u}$ is some extension of $u$ to a three dimensional neighbourhood of $\partial \mathcal{O}$. We denote their adjoints $-\operatorname{div}_{\partial \mathcal{O}}$ : $\boldsymbol{L}_{t}^{2}(\partial \mathcal{O}) \rightarrow H^{-1}(\partial \mathcal{O})$ and $\operatorname{curl}_{\partial \mathcal{O}}: \boldsymbol{L}_{t}^{2}(\partial \mathcal{O}) \rightarrow H^{-1}(\partial \mathcal{O})$ that are defined for all $u \in H^{1}(\partial \mathcal{O})$ and $\mathbf{v} \in \boldsymbol{L}_{t}^{2}(\partial \mathcal{O})$ by

$$
\begin{gathered}
\int_{\partial \mathcal{O}} \nabla_{\partial \mathcal{O}} u \cdot \overline{\mathbf{v}} \mathrm{d} s=-\langle u, \operatorname{div} \partial \mathcal{O} \mathbf{v}\rangle_{H^{1}(\partial \mathcal{O}), H^{-1}(\partial \mathcal{O})}, \\
\int_{\partial \mathcal{O}} \operatorname{curl}_{\partial \mathcal{O}} u \cdot \overline{\mathbf{v}} \mathrm{d} s=\left\langle u, \operatorname{curl}_{\partial \mathcal{O}} \mathbf{v}\right\rangle_{H^{1}(\partial \mathcal{O}), H^{-1}(\partial \mathcal{O})} .
\end{gathered}
$$

The vector operators $\operatorname{curl}_{\partial \mathcal{O}}$ and $\nabla_{\partial \mathcal{O}}$ can be extended to continuous linear operators from $H^{s}(\partial \mathcal{O})$ into $\boldsymbol{H}_{t}^{s-1}(\partial \mathcal{O})$ while the scalar operators $\operatorname{div}_{\partial \mathcal{O}}$ and $\operatorname{curl}_{\partial \mathcal{O}}$ can be extended to continuous linear operators from $\boldsymbol{H}_{t}^{s}(\partial \mathcal{O})$ into $H^{s-1}(\partial \mathcal{O})$ for $s \in[-1 / 2,3 / 2]$. Moreover,

$$
\operatorname{curl}_{\partial \mathcal{O}} \mathbf{u}:=\boldsymbol{\nu} \cdot \operatorname{curl} \widetilde{\mathbf{u}} \text { and } \operatorname{div}_{\partial \mathcal{O}} \mathbf{u}=\operatorname{curl}_{\partial \mathcal{O}}(\boldsymbol{\nu} \times \mathbf{u})
$$

for all $\mathbf{u} \in \boldsymbol{H}_{t}^{1}(\partial \mathcal{O})$ where $\widetilde{\mathbf{u}}$ is some extension of $\mathbf{u}$ to a neighbourhood of $\partial \mathcal{O}$.

We conclude this section by introducing the following boundary spaces for $s \in[-1 / 2,1 / 2]$ :

$$
\begin{aligned}
\boldsymbol{H}^{s}\left(\operatorname{div}_{\partial \mathcal{O}}, \partial \mathcal{O}\right) & :=\left\{\mathbf{v} \in \boldsymbol{H}_{t}^{s}(\partial \mathcal{O}) \mid \operatorname{div}_{\partial \mathcal{O}} \mathbf{v} \in H^{s}(\partial \mathcal{O})\right\}, \\
\boldsymbol{H}^{s}\left(\operatorname{curl}_{\partial \mathcal{O}}, \partial \mathcal{O}\right) & :=\left\{\mathbf{v} \in \boldsymbol{H}_{t}^{s}(\partial \mathcal{O}) \mid \operatorname{curl}_{\partial \mathcal{O}} \mathbf{v} \in H^{s}(\partial \mathcal{O})\right\} .
\end{aligned}
$$

The specific spaces $\boldsymbol{H}^{-1 / 2}\left(\operatorname{curl}_{\partial \mathcal{O}}, \partial \mathcal{O}\right)$ and $\boldsymbol{H}^{-1 / 2}\left(\operatorname{div}_{\partial \mathcal{O}}, \partial \mathcal{O}\right)$ are dual to each other with $\boldsymbol{L}_{t}^{2}(\partial \mathcal{O})$ as pivot space and the trace operators

$$
\gamma_{t}: \boldsymbol{H}(\operatorname{curl}, \mathcal{O}) \rightarrow \boldsymbol{H}^{-1 / 2}\left(\operatorname{div}_{\partial \mathcal{O}}, \partial \mathcal{O}\right), \quad \gamma_{T}: \boldsymbol{H}(\operatorname{curl}, \mathcal{O}) \rightarrow \boldsymbol{H}^{-1 / 2}\left(\operatorname{curl}_{\partial \mathcal{O}}, \partial \mathcal{O}\right)
$$

are linear continuous and surjective. Moreover, we recall that the following formula holds for $\mathbf{u}$ and $\mathbf{v}$ in $\boldsymbol{H}(\operatorname{curl}, \mathcal{O})$ :

$$
\int_{\mathcal{O}}(\operatorname{curl} \mathbf{u} \cdot \overline{\mathbf{v}}-\mathbf{u} \cdot \operatorname{curl} \overline{\mathbf{v}}) \mathrm{d} x=\left\langle\gamma_{t}(\mathbf{u}), \gamma_{T}(\mathbf{v})\right\rangle_{\boldsymbol{H}^{-1 / 2}\left(\operatorname{div}_{\partial \mathcal{O}}, \partial \mathcal{O}\right), \boldsymbol{H}^{-1 / 2}\left(\operatorname{curl}_{\partial \mathcal{O}}, \partial \mathcal{O}\right)} .
$$

See for example ([15], Thm. 3.31). 


\section{Study of AN ABSTRACT Boundary VAlue PROBlem}

Let us denote by $\mathbf{V}(\Gamma) \subset \boldsymbol{L}_{t}^{2}(\Gamma)$ endowed with its inner product $(\cdot, \cdot)_{\mathbf{V}(\Gamma)}$ a Hilbert space that is such that

$$
\left\{\mathbf{v} \in\left(C^{\infty}(\Gamma)\right)^{3} \mid \mathbf{v} \cdot \boldsymbol{\nu}=0\right\} \subset \mathbf{V}(\Gamma)
$$

and such that the injection is dense. Let us denote $\mathbf{V}(\Gamma)^{*}$ the dual space of $\mathbf{V}(\Gamma)$ with respect to $\boldsymbol{L}_{t}^{2}(\Gamma)$. The impedance operator is defined as follows.

Definition 3.1. A generalized impedance operator $\mathcal{Z}$ is a linear and bounded operator from $\mathbf{V}(\Gamma)$ into its dual $\mathbf{V}(\Gamma)^{*}$.

We define $\boldsymbol{H}_{\text {loc }}\left(\mathbf{c u r l}, \Omega_{\text {ext }}\right)$ as being the set of functions in $\boldsymbol{H}\left(\mathbf{c u r l}, B_{R} \backslash \bar{\Omega}\right)$ for every ball $B_{R}$ of radius $R$ that contains $\bar{\Omega}$ and $V_{\mathbf{H}}:=\left\{\mathbf{H} \in \boldsymbol{H}_{\mathrm{loc}}\left(\mathbf{c u r l}, \Omega_{\mathrm{ext}}\right) \mid \mathbf{H}_{T} \in \mathbf{V}(\Gamma)\right\}$, the exterior problem (2.1) together with the radiation condition (2.2) then writes for $\mathbf{f} \in \mathbf{V}(\Gamma)^{*}$ :

$$
\left\{\begin{array}{l}
\text { Find }(\mathbf{E}, \mathbf{H}) \in \boldsymbol{H}_{\mathrm{loc}}\left(\mathbf{c u r l}, \Omega_{\mathrm{ext}}\right) \times V_{\mathbf{H}} \text { such that } \\
\operatorname{curl} \mathbf{H}+i \kappa \epsilon \mathbf{E}=0 \text { in } \Omega_{\mathrm{ext}}, \\
\operatorname{curl} \mathbf{E}-i \kappa \mu \mathbf{H}=0 \quad \text { in } \Omega_{\mathrm{ext}}, \\
\boldsymbol{\nu} \times \mathbf{E}+\mathcal{Z} \mathbf{H}_{T}=\mathbf{f} \text { on } \Gamma, \\
\lim _{R \rightarrow \infty}|\mathbf{H} \times \hat{x}-(\hat{x} \times \mathbf{E}) \times \hat{x}|^{2} \mathrm{~d} s=0 .
\end{array}\right.
$$

To study existence and uniqueness of the solution to (3.1) we have to reformulate these equations in a bounded domain. In the following we propose two different approaches to achieve this goal. The first is rather classical and consists in bounding the domain $\Omega_{\text {ext }}$ by introducing a ball that contains the domain $\Omega$ and by applying a transparent boundary condition on this artificial boundary. The second approach consist in writing the system (3.1) as a single equation on $\Gamma$ by using the so-called Magnetic-to-Electric Calderón operator for the exterior problem. In Lemma 3.2 we prove that these two formulations are equivalent.

\subsection{A volume formulation in a bounded domain}

Let $B_{R}$ be a ball of radius $R>R_{0}$ such that $\Omega \subset B_{R}$, and let us introduce the Magnetic-to-Electric Calderón operator $\boldsymbol{S}_{R}: \boldsymbol{H}^{-1 / 2}\left(\operatorname{div}_{\partial B_{R}}, \partial B_{R}\right) \rightarrow \boldsymbol{H}^{-1 / 2}\left(\operatorname{div}_{\partial B_{R}}, \partial B_{R}\right)$ defined for $\mathbf{v} \in \boldsymbol{H}^{-1 / 2}\left(\operatorname{div}_{\partial B_{R}}, \partial B_{R}\right)$ by $\boldsymbol{S}_{R} \mathbf{v}:=\hat{x} \times \mathbf{E}$ where $(\mathbf{E}, \mathbf{H}) \in \boldsymbol{H}_{\text {loc }}\left(\operatorname{curl}, \mathbb{R}^{3} \backslash \overline{B_{R}}\right) \times \boldsymbol{H}_{\text {loc }}\left(\mathbf{c u r l}, \mathbb{R}^{3} \backslash \overline{B_{R}}\right)$ is the unique solution (see [8] for fundamental results about electromagnetic scattering theory) to

$$
\left\{\begin{array}{l}
\operatorname{curl} \mathbf{H}+i \kappa \mathbf{E}=0 \quad \text { in } \mathbb{R}^{3} \backslash \overline{B_{R}}, \\
\operatorname{curl} \mathbf{E}-i \kappa \mathbf{H}=0 \quad \text { in } \mathbb{R}^{3} \backslash \overline{B_{R}}, \\
\hat{x} \times \mathbf{H}=\mathbf{v} \quad \text { on } \partial B_{R}, \\
\lim _{r \rightarrow \infty} \int_{\partial B_{r}}|\mathbf{H} \times \hat{x}-(\hat{x} \times \mathbf{E}) \times \hat{x}|^{2} \mathrm{~d} s=0 .
\end{array}\right.
$$

Let us denote $\Omega_{R}:=B_{R} \backslash \bar{\Omega}$ and let us define the Hilbert space $V_{\mathbf{H}, R}:=\left\{\mathbf{v} \in \boldsymbol{H}\left(\operatorname{curl}, \Omega_{R}\right) \mid \mathbf{v}_{T} \in \mathbf{V}(\Gamma)\right\}$ endowed with the norm

$$
\|\cdot\|_{V_{\mathbf{H}, R}}:=\|\cdot\|_{\boldsymbol{H}\left(\operatorname{curl}, \Omega_{R}\right)}+\|\cdot\|_{\mathbf{V}(\Gamma)} .
$$

Then, for any $\mathbf{f} \in \mathbf{V}(\Gamma)^{*}$, problem (3.1) is equivalent to:

$$
\left\{\begin{array}{l}
\text { Find }(\mathbf{E}, \mathbf{H}) \in \mathbf{H}\left(\operatorname{curl}, \Omega_{R}\right) \times V_{\mathbf{H}, R} \text { such that } \\
\operatorname{curl} \mathbf{H}+i \kappa \epsilon \mathbf{E}=0 \text { in } \Omega_{R}, \\
\operatorname{curl} \mathbf{E}-i \kappa \mu \mathbf{H}=0 \text { in } \Omega_{R}, \\
\boldsymbol{\nu} \times \mathbf{E}+\mathcal{Z} \mathbf{H}_{T}=\mathbf{f} \text { on } \Gamma, \\
\hat{x} \times \mathbf{E}-\boldsymbol{S}_{R}(\hat{x} \times \mathbf{H})=0 \text { on } \partial B_{R}
\end{array}\right.
$$


THE ELECTROMAGNETIC SCATTERING PROBLEM WITH GENERALIZED IMPEDANCE BOUNDARY CONDITIONS 909

which is equivalent to find $\mathbf{H} \in V_{\mathbf{H}, R}$ such that

$$
\begin{aligned}
& \int_{\Omega_{R}} \epsilon^{-1} \operatorname{curl} \mathbf{H} \cdot \operatorname{curl} \overline{\mathbf{v}}-\kappa^{2} \mu \mathbf{H} \cdot \overline{\mathbf{v}} \mathrm{d} x+i \kappa\langle\mathcal{Z} \mathbf{H}, \mathbf{v}\rangle_{\mathbf{V}(\Gamma)^{*}, \mathbf{V}(\Gamma)}-i \kappa \int_{\partial B_{R}} \boldsymbol{S}_{R}(\hat{x} \times \mathbf{H}) \cdot \overline{\mathbf{v}} \mathrm{d} s \\
& =i \kappa\langle\mathbf{f}, \mathbf{v}\rangle_{\mathbf{V}(\Gamma)^{*}, \mathbf{V}(\Gamma)}
\end{aligned}
$$

for all $\mathbf{v} \in V_{\mathbf{H}, R}$. To ensure weak coercivity of this variational formulation one has to assume that the imaginary part of $\mathcal{Z}$ is negative. In fact, this is not always necessary and we overcome this difficulty by introducing an alternative formulation for problem (3.1) in next section.

\subsection{A surface formulation}

Let us introduce the so-called Magnetic-to-Electric Calderón operator $\boldsymbol{S}_{\Gamma}: \boldsymbol{H}^{-1 / 2}\left(\operatorname{curl}_{\Gamma}, \Gamma\right) \rightarrow$ $\boldsymbol{H}^{-1 / 2}\left(\operatorname{div}_{\Gamma}, \Gamma\right)$ defined for $\mathbf{v} \in \boldsymbol{H}^{-1 / 2}\left(\operatorname{curl}_{\Gamma}, \Gamma\right)$ by $\boldsymbol{S}_{\Gamma} \mathbf{v}:=\boldsymbol{\nu} \times \mathbf{E}$ where $(\mathbf{E}, \mathbf{H}) \in \boldsymbol{H}_{\text {loc }}\left(\operatorname{curl}, \Omega_{\mathrm{ext}}\right) \times$ $\boldsymbol{H}_{\text {loc }}\left(\mathbf{c u r l}, \Omega_{\text {ext }}\right)$ is the unique solution to

$$
\left\{\begin{array}{l}
\operatorname{curl} \mathbf{H}+i \kappa \epsilon \mathbf{E}=0 \quad \text { in } \Omega_{\mathrm{ext}}, \\
\operatorname{curl} \mathbf{E}-i \kappa \mu \mathbf{H}=0 \quad \text { in } \Omega_{\mathrm{ext}}, \\
\mathbf{H}_{T}=\mathbf{v} \quad \text { on } \Gamma, \\
\lim _{R \rightarrow \infty} \int_{\partial B_{R}}|\mathbf{H} \times \hat{x}-(\hat{x} \times \mathbf{E}) \times \hat{x}|^{2} \mathrm{~d} s=0
\end{array}\right.
$$

and we recall that this operator is linear and continuous (see [15] Chap. 10, for more details). Using $\boldsymbol{S}_{\Gamma}$, problem (3.1) can be rewritten in these terms:

$$
\left\{\begin{array}{l}
\text { Find } \mathbf{u} \in \mathbf{V}(\Gamma) \cap \boldsymbol{H}^{-1 / 2}\left(\operatorname{curl}_{\Gamma}, \Gamma\right) \text { such that } \\
\boldsymbol{S}_{\Gamma}(\mathbf{u})+\mathcal{Z} \mathbf{u}=\mathbf{f}
\end{array}\right.
$$

for $\mathbf{f} \in\left(\mathbf{V}(\Gamma) \cap \boldsymbol{H}^{-1 / 2}\left(\operatorname{curl}_{\Gamma}, \Gamma\right)\right)^{*}$ where $\mathbf{V}(\Gamma) \cap \boldsymbol{H}^{-1 / 2}\left(\operatorname{curl}_{\Gamma}, \Gamma\right)$ is endowed with the norm

$$
\|\cdot\|_{\mathbf{V}(\Gamma) \cap \boldsymbol{H}^{-1 / 2}\left(\operatorname{curl}_{\Gamma}, \Gamma\right)}:=\|\cdot\|_{\mathbf{V}(\Gamma)}+\|\cdot\|_{\boldsymbol{H}^{-1 / 2}\left(\operatorname{curl}_{\Gamma}, \Gamma\right)} .
$$

Equation (3.5) makes sense in $\left(\mathbf{V}(\Gamma) \cap \boldsymbol{H}^{-1 / 2}\left(\operatorname{curl}_{\Gamma}, \Gamma\right)\right)^{*}$ since $\boldsymbol{S}_{\Gamma}(\mathbf{u}) \in \boldsymbol{H}_{\operatorname{div}_{\Gamma}}^{-1 / 2}(\Gamma)$ and this space can be identified with the dual space of $\boldsymbol{H}^{-1 / 2}\left(\operatorname{curl}_{\Gamma}, \Gamma\right)$. Therefore, $\boldsymbol{S}_{\Gamma}(\mathbf{u})$ can be identified with a linear and continuous application on $\boldsymbol{H}^{-1 / 2}\left(\operatorname{curl}_{\Gamma}, \Gamma\right)$ and consequently on $\left(\mathbf{V}(\Gamma) \cap \boldsymbol{H}^{-1 / 2}\left(\operatorname{curl}_{\Gamma}, \Gamma\right)\right)$. As stated in the next Lemma, problems (3.1) and (3.5) are equivalent.

Lemma 3.2. Let $\boldsymbol{f}$ be in $\left(\boldsymbol{V}(\Gamma) \cap \mathbf{H}^{-1 / 2}\left(\operatorname{curl}_{\Gamma}, \Gamma\right)\right)^{*}$, if $\boldsymbol{u} \in \boldsymbol{V}(\Gamma) \cap \mathbf{H}^{-1 / 2}\left(\operatorname{curl}_{\Gamma}, \Gamma\right)$ solves (3.5) then the unique solution $(\boldsymbol{E}, \boldsymbol{H}) \in \mathbf{H}_{l o c}\left(\mathbf{c u r l}, \Omega_{\mathrm{ext}}\right) \times V_{\boldsymbol{H}}$ to (3.4) with $\boldsymbol{v}=\boldsymbol{u}$ solves (3.1). Conversely, if $(\boldsymbol{E}, \boldsymbol{H}) \in$ $\mathbf{H}_{l o c}\left(\mathbf{c u r l}, \Omega_{\mathrm{ext}}\right) \times V_{\boldsymbol{H}}$ solves (3.1) then $\boldsymbol{H}_{T} \in \boldsymbol{V}(\Gamma) \cap \mathbf{H}^{-1 / 2}\left(\operatorname{curl}_{\Gamma}, \Gamma\right)$ and solves (3.5).

Proof. We take $f \in\left(\mathbf{V}(\Gamma) \cap \boldsymbol{H}^{-1 / 2}\left(\operatorname{curl}_{\Gamma}, \Gamma\right)\right)^{*}$ and let $\mathbf{u} \in \mathbf{V}(\Gamma) \cap \boldsymbol{H}^{-1 / 2}\left(\operatorname{curl}_{\Gamma}, \Gamma\right)$ be a solution to (3.5). We define $(\mathbf{E}, \mathbf{H}) \in \boldsymbol{H}_{\text {loc }}\left(\mathbf{c u r l}, \Omega_{\text {ext }}\right) \times V_{\mathbf{H}}$ as being the unique solution to (3.4) for $\mathbf{v}=\mathbf{u}$ on $\Gamma$. The tangential component of $\mathbf{H}$ satisfies $\mathbf{H}_{T}=\mathbf{u}$ on $\Gamma$ and then $\mathbf{H} \in V_{\mathbf{H}}$. Finally, since $\boldsymbol{\nu} \times \mathbf{E}=\boldsymbol{S}_{\Gamma}(\mathbf{u})$ and since $\mathbf{u}$ solves (3.5) we obtain

$$
\boldsymbol{\nu} \times \mathbf{E}+\mathcal{Z} \mathbf{H}_{T}=f \quad \text { on } \Gamma
$$

which means that $(\mathbf{E}, \mathbf{H})$ solves (3.1).

The reverse statement is straightforward since $\boldsymbol{\nu} \times \mathbf{E}=\boldsymbol{S}_{\Gamma}\left(\mathbf{H}_{T}\right)$ as soon as $(\mathbf{E}, \mathbf{H})$ solves Maxwell's equations outside $\Omega$.

We therefore obtain that problems (3.1), (3.2) and (3.5) are equivalent. We establish now a well-posedness result for $(3.1)$ which is valid for a general class of operators $\mathcal{Z}$. 


\subsection{Existence and uniqueness for a general class of boundary conditions}

First, to ensure uniqueness, we impose a certain absorption condition to be satisfied by the boundary operator $\mathcal{Z}$. In general this hypothesis is not restrictive since it is linked to some absorption property of the modelled material.

Hypothesis 3.3. The operator $\mathcal{Z}$ has a nonpositive real part, that is:

$$
\Re\langle\mathcal{Z} \boldsymbol{v}, \boldsymbol{v}\rangle_{\boldsymbol{V}(\Gamma)^{*}, \boldsymbol{V}(\Gamma)} \leq 0
$$

for all $\boldsymbol{v} \in \boldsymbol{V}(\Gamma)$.

Under this hypothesis we prove uniqueness.

Theorem 3.4. If Hypothesis 3.3 is satisfied then problem (3.1) has at most one solution.

Proof. Assume that $(\mathbf{E}, \mathbf{H}) \in \boldsymbol{H}_{\text {loc }}\left(\mathbf{c u r l}, \Omega_{\text {ext }}\right) \times V_{\mathbf{H}}$ satisfies (3.1) with $\mathbf{f}=0$ on $\Gamma$. Let $B_{R}$ be a ball of radius $R>R_{0}$ that contains $\bar{\Omega}$, by using the integration by part formula (2.4) in $\Omega_{\text {ext }} \cap B_{R}$ we find that $(\mathbf{E}, \mathbf{H})$ satisfies

$$
\begin{aligned}
\left\langle\gamma_{t} \mathbf{E}, \gamma_{T} \mathbf{H}\right\rangle_{\boldsymbol{H}^{-1 / 2}\left(\operatorname{div}_{\Gamma}, \Gamma\right), \boldsymbol{H}^{-1 / 2}\left(\operatorname{curl}_{\Gamma}, \Gamma\right)}+\int_{\partial B_{R}} \hat{x} \times \mathbf{E} \cdot \overline{\mathbf{H}} \mathrm{d} s & =\int_{\Omega_{\mathrm{ext}^{\prime} \cap B_{R}}} \operatorname{curl} \mathbf{E} \cdot \overline{\mathbf{H}}-\mathbf{E} \cdot \operatorname{curl} \overline{\mathbf{H}} \mathrm{d} x \\
& =\int_{\Omega_{\mathrm{ext}} \cap B_{R}} i \kappa \mu|\mathbf{H}|^{2}+\overline{i \kappa \epsilon}|\mathbf{E}|^{2} \mathrm{~d} x .
\end{aligned}
$$

By taking the real part of this equality we obtain

$$
\begin{aligned}
\Re\left\langle\gamma_{t} \mathbf{E}, \gamma_{T} \mathbf{H}\right\rangle_{H^{-1 / 2}\left(\operatorname{div}_{\Gamma}, \Gamma\right), \boldsymbol{H}^{-1 / 2}\left(\operatorname{curl}_{\Gamma}, \Gamma\right)}=-\Re & \left(\int_{\partial B_{R}} \hat{x} \times \mathbf{E} \cdot \overline{\mathbf{H}} \mathrm{d} s\right) \\
& +\int_{\Omega_{\mathrm{ext}} \cap B_{R}} \Re(i \kappa \mu)|\mathbf{H}|^{2}+\Re(i \kappa \epsilon)|\mathbf{E}|^{2} \mathrm{~d} s
\end{aligned}
$$

and since $\boldsymbol{\nu} \times \mathbf{E}=-\mathcal{Z} \mathbf{H}_{T}$ on $\Gamma$ this relation becomes

$$
\Re\left\langle\mathcal{Z} \mathbf{H}_{T}, \mathbf{H}_{T}\right\rangle_{\mathbf{V}(\Gamma)^{*}, \mathbf{V}(\Gamma)}=\Re\left(\int_{\partial B_{R}} \hat{x} \times \mathbf{E} \cdot \overline{\mathbf{H}} \mathrm{d} s\right)-\int_{\Omega_{\mathrm{ext}} \cap B_{R}} \Re(i \kappa \mu)|\mathbf{H}|^{2}+\Re(i \kappa \epsilon)|\mathbf{E}|^{2} \mathrm{~d} s .
$$

Since we assume that the real part of $\mathcal{Z}$ is nonpositive and since the imaginary parts of $\epsilon$ and $\mu$ are nonnegative, this gives

$$
\Re\left(\int_{\partial B_{R}} \hat{x} \times \mathbf{E} \cdot \overline{\mathbf{H}} \mathrm{d} s\right) \leq 0
$$

which in regards of Rellich's Lemma ([15], Lem. 9.28) gives $\mathbf{E}=\mathbf{H}=0$ in $\mathbb{R}^{3} \backslash \overline{B_{R}}$. Since the coefficients $\epsilon$ and $\mu$ are continuously differentiable, $\mathbf{E}$ and $\mathbf{H}$ are in $\left(H^{1}(\mathcal{O})\right)^{3}$ for all open set $\mathcal{O}$ such that $\overline{\mathcal{O}} \subset \Omega_{\text {ext }}$ and the unique continuation principle given in [17] applies. That gives $\mathbf{E}=\mathbf{H}=0$ in $\Omega_{\text {ext }}$ and it concludes the proof.

As a consequence, to prove that problem (3.1) is well-posed it is sufficient to prove that it can be formulated as a Fredholm type problem. When $\mathbf{V}(\Gamma)$ is compactly embedded into $\boldsymbol{H}^{-1 / 2}\left(\operatorname{curl}_{\Gamma}, \Gamma\right)$ the surface formulation (3.5) allows to prove this property in a straightforward way as soon as $\mathcal{Z}: \mathbf{V}(\Gamma) \rightarrow \mathbf{V}(\Gamma)^{*}$ can be decomposed as the sum of an isomorphism and a compact operator.

Theorem 3.5. Let $\mathcal{Z}$ be an impedance operator such that Hypothesis 3.3 is satisfied. If $\boldsymbol{V}(\Gamma)$ is compactly embedded into $\mathbf{H}^{-1 / 2}\left(\operatorname{curl}_{\Gamma}, \Gamma\right)$ and $\mathcal{Z}=\mathcal{T}+\mathcal{K}$ where $\mathcal{T}: \boldsymbol{V}(\Gamma) \rightarrow \boldsymbol{V}(\Gamma)^{*}$ is an isomorphism and $\mathcal{K}: \boldsymbol{V}(\Gamma) \rightarrow$ $\boldsymbol{V}(\Gamma)^{*}$ is a bounded and compact operator, then for all $\boldsymbol{f} \in \boldsymbol{V}(\Gamma)^{*}$ problem (3.1) has a unique solution $(\boldsymbol{E}, \boldsymbol{H}) \in$ $\mathbf{H}_{l o c}\left(\mathbf{c u r l}, \Omega_{\mathrm{ext}}\right) \times V_{\boldsymbol{H}}$ and for every ball $B_{R}$ that contains $\bar{\Omega}$ it exists $C_{R}>0$ such that

$$
\|\boldsymbol{E}\|_{\mathbf{H}\left(\operatorname{curl}, \Omega_{R}\right)}+\|\boldsymbol{H}\|_{V_{\boldsymbol{H}, R}} \leq C_{R}\|\boldsymbol{f}\|_{\boldsymbol{V}(\Gamma)^{*}}
$$


THE ELECTROMAGNETIC SCATTERING PROBLEM WITH GENERALIZED IMPEDANCE BOUNDARY CONDITIONS 911

Proof. First of all, $\mathbf{V}(\Gamma) \cap \boldsymbol{H}^{-1 / 2}\left(\operatorname{curl}_{\Gamma}, \Gamma\right)=\mathbf{V}(\Gamma)$ with equivalence of norms and we have equivalence between (3.1) and (3.5) in the sense of Lemma 3.2. Let us prove that $\boldsymbol{S}_{\Gamma}+\mathcal{Z}: \mathbf{V}(\Gamma) \rightarrow \mathbf{V}(\Gamma)^{*}$ is of Fredholm type. The operator $\boldsymbol{S}_{\Gamma}: \boldsymbol{H}^{-1 / 2}\left(\operatorname{curl}_{\Gamma}, \Gamma\right) \rightarrow \mathbf{V}(\Gamma)^{*}$ is continuous and therefore is compact from $\mathbf{V}(\Gamma)$ into $\mathbf{V}(\Gamma)^{*}$ since we assumed that $\mathbf{V}(\Gamma)$ is compactly embedded into $\boldsymbol{H}^{-1 / 2}\left(\operatorname{curl}_{\Gamma}, \Gamma\right)$. Moreover, $\mathcal{Z}=\mathcal{T}+\mathcal{K}$ with $\mathcal{T}: \mathbf{V}(\Gamma) \rightarrow \mathbf{V}(\Gamma)^{*}$ an isomorphism and $\mathcal{K}: \mathbf{V}(\Gamma) \rightarrow \mathbf{V}(\Gamma)^{*}$ a compact operator and consequently, $\boldsymbol{S}_{\Gamma}+\mathcal{Z}$ is of Fredholm type with index 0. Theorem 3.4 allows to finish the proof.

When $\mathbf{V}(\Gamma)$ is not included into $\boldsymbol{H}^{-1 / 2}\left(\operatorname{curl}_{\Gamma}, \Gamma\right)$, there is no real advantage in using the surface formulation to establish existence and uniqueness of the solution to the scattering problem and we have to impose some restrictions on the sign of the imaginary part of the boundary operator to obtain the following theorem.

Theorem 3.6. Let $\mathcal{Z}$ be an impedance operator such that Hypothesis 3.3 is satisfied and such that it exists $c>0$ such that

$$
\Re\langle i \kappa \mathcal{Z} \boldsymbol{u}, \boldsymbol{u}\rangle_{\boldsymbol{V}(\Gamma)^{*}, \boldsymbol{V}(\Gamma)}>c\|\boldsymbol{u}\|_{\boldsymbol{V}(\Gamma)}^{2} \quad \forall \boldsymbol{u} \in \boldsymbol{V}(\Gamma) .
$$

Then for all $\boldsymbol{f} \in \boldsymbol{V}(\Gamma)^{*}$ problem (3.1) has a unique solution $(\boldsymbol{E}, \boldsymbol{H}) \in \mathbf{H}_{\text {loc }}\left(\mathbf{c u r l}, \Omega_{\mathrm{ext}}\right) \times V_{\boldsymbol{H}}$ and for every ball $B_{R}$ that contains $\bar{\Omega}$ it exists $C_{R}>0$ such that

$$
\|\boldsymbol{E}\|_{\mathbf{H}\left(\operatorname{curl}, \Omega_{R}\right)}+\|\boldsymbol{H}\|_{V_{H, R}} \leq C_{R}\|\boldsymbol{f}\|_{\boldsymbol{V}(\Gamma)^{*}}
$$

Proof. The proof of this result is a slight adaptation of the procedure presented in ([15], Chap. 10) and is therefore postponed in appendix.

\section{WELL-POSEDNESS FOR SECOND ORDER SURFACE DIFFERENTIAL OPERATORS}

In this section we will consider three different second order surface differential operators and we will see that these operators are very different from one another and require different treatments. For the first two cases we will prove the Fredholm property of the surface formulation (3.5) and use Theorem 3.5 while in the third case $\mathbf{V}(\Gamma)$ is not a subspace of $\boldsymbol{H}^{-1 / 2}\left(\operatorname{curl}_{\Gamma}, \Gamma\right)$ and we will make use of Theorem 3.6. While the case treated in Section 4.2 corresponds to an approximate model for thin layers (see [11] for example), the two other cases (Sects. 4.3 and 4.1) are presented here mainly for their theoretical interest. To the knowledge of the author, they are not known yet to be approximate models of a given physical configuration.

\subsection{The case of $\mathcal{Z}=\operatorname{curl}_{\Gamma} \eta \operatorname{curl}_{\Gamma}+\nabla_{\Gamma} \gamma \operatorname{div}_{\Gamma}+\lambda$}

We take $(\lambda, \eta, \gamma) \in\left(L^{\infty}(\Gamma)\right)^{3}$ and we define

$$
\mathcal{Z}=\operatorname{curl}_{\Gamma} \eta \operatorname{curl}_{\Gamma}+\nabla_{\Gamma} \gamma \operatorname{div}_{\Gamma}+\lambda
$$

which is a bounded and linear operator from $\mathbf{V}(\Gamma)=\boldsymbol{H}^{0}\left(\operatorname{div}_{\Gamma}, \Gamma\right) \cap \boldsymbol{H}^{0}\left(\operatorname{curl}_{\Gamma}, \Gamma\right)$ into its dual. For all $\mathbf{v}, \mathbf{w}$ in $\mathbf{V}(\Gamma)$ we have

$$
\langle\mathcal{Z} \mathbf{v}, \mathbf{w}\rangle_{\mathbf{V}(\Gamma)^{*}, \mathbf{V}(\Gamma)}:=\int_{\Gamma} \eta \operatorname{curl}_{\Gamma} \mathbf{v} \overline{\operatorname{curl}_{\Gamma} \mathbf{w}}-\gamma \operatorname{div}_{\Gamma} \mathbf{v} \overline{\operatorname{div}_{\Gamma} \mathbf{w}}+\lambda \mathbf{v} \cdot \overline{\mathbf{w}} \mathrm{d} s .
$$

The space $\mathbf{V}(\Gamma)$ is endowed with the norm

$$
\|\cdot\|_{\mathbf{V}(\Gamma)}:=\|\cdot\|_{\boldsymbol{H}^{0}\left(\operatorname{div}_{\Gamma}, \Gamma\right)}+\|\cdot\|_{\boldsymbol{H}^{0}\left(\operatorname{curl}_{\Gamma}, \Gamma\right)}
$$

and this space is nothing but $\boldsymbol{H}_{t}^{1}(\Gamma)$ since we have the algebraic relation

$$
-\Delta_{\Gamma}=\operatorname{curl}_{\Gamma} \operatorname{curl}_{\Gamma}-\nabla_{\Gamma} \operatorname{div}_{\Gamma}
$$

where $\boldsymbol{\Delta}_{\Gamma}$ is the vector Laplace Beltrami operator on $\Gamma$. As a consequence, the embedding of $\mathbf{V}(\Gamma)$ in $\boldsymbol{H}^{-1 / 2}\left(\operatorname{curl}_{\Gamma}, \Gamma\right)$ and $\boldsymbol{L}_{t}^{2}(\Gamma)$ is compact. Therefore we can use the surface formulation (3.5) to prove that problem (3.1) is well-posed under the following sign assumptions on $\lambda, \eta$ and $\gamma$. 
Hypothesis 4.1. The functions $(\lambda, \eta, \gamma) \in\left(L^{\infty}(\Gamma)\right)^{3}$ are such that

$$
\Re(\lambda) \leq 0, \quad \Re(\eta) \leq 0, \quad \Re(\gamma) \geq 0 \quad \text { a.e. on } \Gamma
$$

it exists $c>0$ such that

$$
|\gamma| \geq c, \quad|\eta| \geq c \quad \text { a.e. on } \Gamma
$$

and the imaginary parts of $\gamma$ and $\eta$ do not change sign on $\Gamma$ and are of opposite sign.

The following theorem is then a consequence of Theorem 3.5.

Theorem 4.2. If $(\lambda, \eta, \gamma)$ satisfy Hypothesis 4.1 then for all $\boldsymbol{f} \in \boldsymbol{V}(\Gamma)^{*}$ problem (3.1) with $\mathcal{Z}=\operatorname{curl}_{\Gamma} \eta \operatorname{curl}_{\Gamma}+$ $\nabla_{\Gamma} \gamma \operatorname{div}_{\Gamma}+\lambda$ has a unique solution $(\boldsymbol{E}, \boldsymbol{H})$ and for every ball $B_{R}$ that contains $\bar{\Omega}$ it exists $C_{R}>0$ such that

$$
\|\boldsymbol{E}\|_{\mathbf{H}\left(\operatorname{curl}, \Omega_{R}\right)}+\|\boldsymbol{H}\|_{V_{H, R}} \leq C_{R}\|\boldsymbol{f}\|_{V(\Gamma)^{*}} .
$$

Proof. Let us assume that $(\lambda, \eta, \gamma) \in\left(L^{\infty}(\Gamma)\right)^{3}$ satisfy Hypothesis 4.1, then the real part of $\mathcal{Z}$ is nonpositive and from Theorem 3.4 we deduce that problem (3.1) with $\mathcal{Z}=\operatorname{curl}_{\Gamma} \eta \operatorname{curl}{ }_{\Gamma}+\nabla_{\Gamma} \gamma \operatorname{div}_{\Gamma}+\lambda$ has at most one solution. To prove existence we use the surface formulation (3.5) which is equivalent to (3.1) since $\mathbf{V}(\Gamma) \subset$ $\boldsymbol{H}^{-1 / 2}\left(\operatorname{curl}_{\Gamma}, \Gamma\right)$. Let us define the bounded linear operators $T: \mathbf{V}(\Gamma) \rightarrow \mathbf{V}(\Gamma)^{*}$ and $K: \mathbf{V}(\Gamma) \rightarrow \mathbf{V}(\Gamma)^{*}$ by

$$
\begin{aligned}
\langle T \mathbf{v}, \mathbf{w}\rangle_{\mathbf{V}(\Gamma)^{*}, \mathbf{V}(\Gamma)}:= & \int_{\Gamma} \eta \operatorname{curl}_{\Gamma} \mathbf{v} \operatorname{curl}_{\Gamma} \overline{\mathbf{w}} \mathrm{d} s-\int_{\Gamma} \gamma \operatorname{div}_{\Gamma} \mathbf{v} \operatorname{div}_{\Gamma} \overline{\mathbf{w}} \mathrm{d} s+\int_{\Gamma} \eta \mathbf{v} \cdot \overline{\mathbf{w}} \mathrm{d} s, \\
& \langle K \mathbf{v}, \mathbf{w}\rangle_{\mathbf{V}(\Gamma)^{*}, \mathbf{V}(\Gamma)}:=\int_{\Gamma}(\lambda-\eta) \mathbf{v} \cdot \overline{\mathbf{w}} \mathrm{d} s
\end{aligned}
$$

for all $\mathbf{v}$ and $\mathbf{w}$ in $\mathbf{V}(\Gamma)$ and then $\mathcal{Z}=T+K$. We recall that for any complex number $z=a+i b \in \mathbb{C}$ we have

$$
|z| \geq \frac{|a|+|b|}{\sqrt{2}}
$$

and since $(\lambda, \eta, \gamma)$ satisfy Hypothesis 4.1 , this last inequality implies that the operator $T$ is coercive on $\mathbf{V}(\Gamma)$ i.e. it exists $C>0$ such that

$$
\left|\langle T \mathbf{u}, \mathbf{u}\rangle_{\mathbf{V}(\Gamma)^{*}, \mathbf{V}(\Gamma)}\right| \geq C\|\mathbf{u}\|_{\mathbf{V}(\Gamma)}^{2} \quad \forall \mathbf{u} \in \mathbf{V}(\Gamma) .
$$

Moreover, since the embeddings of $\mathbf{V}(\Gamma)$ into $\boldsymbol{L}_{t}^{2}(\Gamma)$ and $\boldsymbol{H}^{-1 / 2}\left(\operatorname{curl}_{\Gamma}, \Gamma\right)$ are compact, we deduce that $K$ : $\mathbf{V}(\Gamma) \rightarrow \mathbf{V}(\Gamma)^{*}$ and $\boldsymbol{S}_{\Gamma}: \mathbf{V}(\Gamma) \rightarrow \mathbf{V}(\Gamma)^{*}$ are compact operators. Then, Theorem 3.5 concludes the proof.

Remark 4.3. Using formulation (3.5) instead of formulation (3.2) to prove existence and uniqueness of the solution to the scattering problem allows us to treat the case of coefficients $\eta$ and $\gamma$ with negative and positive imaginary parts respectively. This could not be achieved with standard variational arguments on the variational formulation (3.3) associated with the volume problem (3.2).

\subsection{The case of $\mathcal{Z}=\operatorname{curl}_{\Gamma} \eta \operatorname{curl}_{\Gamma}+\lambda$}

Let $\lambda$ and $\eta$ be two $L^{\infty}(\Gamma)$ functions and let us define

$$
\mathcal{Z}=\operatorname{curl}_{\Gamma} \eta \operatorname{curl}_{\Gamma}+\lambda
$$

which is bounded and continuous from $\mathbf{V}(\Gamma)=\boldsymbol{H}^{0}\left(\operatorname{curl}_{\Gamma}, \Gamma\right)$ into its dual. Similarly to the previous case, we have for all $\mathbf{v}, \mathbf{w}$ in $\mathbf{V}(\Gamma)$ :

$$
\langle\mathcal{Z} \mathbf{v}, \mathbf{w}\rangle_{\mathbf{V}(\Gamma)^{*}, \mathbf{V}(\Gamma)}:=\int_{\Gamma} \eta \operatorname{curl}_{\Gamma} \mathbf{v} \overline{\operatorname{curl}_{\Gamma} \mathbf{W}}+\lambda \mathbf{v} \cdot \overline{\mathbf{w}} \mathrm{d} s .
$$

We assume that $\lambda$ and $\eta$ satisfy the following sign hypothesis. 
Hypothesis 4.4. The functions $(\lambda, \eta) \in\left(L^{\infty}(\Gamma)\right)^{2}$ are such that

$$
\Re(\lambda) \leq 0, \quad \Re(\eta) \leq 0 \quad \text { a.e. on } \Gamma,
$$

it exists $c>0$ such that

$$
|\lambda| \geq c, \quad|\eta| \geq c \quad \text { a.e. on } \Gamma
$$

and the imaginary parts of $\lambda$ and $\eta$ do not change sign on $\Gamma$.

Under Hypothesis 4.4, we obtain the following result.

Theorem 4.5. Let $(\lambda, \eta) \in\left(L^{\infty}(\Gamma)\right)^{2}$ be such that Hypothesis 4.4 is satisfied. Then for all $\boldsymbol{f} \in \boldsymbol{V}(\Gamma)^{*}$ problem (3.1) with $\mathcal{Z}=\operatorname{curl}_{\Gamma} \eta \operatorname{curl}_{\Gamma}+\lambda$ has a unique solution $(\boldsymbol{E}, \boldsymbol{H})$ and for every ball $B_{R}$ that contains $\bar{\Omega}$ it exists $C_{R}>0$ such that

$$
\|\boldsymbol{E}\|_{\mathbf{H}\left(\operatorname{curl}, \Omega_{R}\right)}+\|\boldsymbol{H}\|_{V_{H, R}} \leq C_{R}\|\boldsymbol{f}\|_{\boldsymbol{V}(\Gamma)^{*}} .
$$

First of all, if the imaginary parts of $\lambda$ and $\eta$ are of the same sign then the situation is very similar to the one in the previous section. Actually, $\mathbf{V}(\Gamma)$ is compactly embedded into $\boldsymbol{H}^{-1 / 2}\left(\operatorname{curl}_{\Gamma}, \Gamma\right)$ and we can use the surface formulation (3.5) to prove that problem (3.1) is well-posed. Indeed, in this case $\mathcal{Z}: \mathbf{V}(\Gamma) \rightarrow \mathbf{V}(\Gamma)^{*}$ is coercive and since $\boldsymbol{S}_{\Gamma}: \mathbf{V}(\Gamma) \rightarrow \mathbf{V}(\Gamma)^{*}$ is compact we deduce well-posedness from the uniqueness Theorem 3.4.

If this is not the case, that is if the imaginary parts of $\lambda$ and $\eta$ are of opposite sign then we have to be much more careful to prove existence of a solution to (3.1) (uniqueness is ensured by Thm. 3.4). As mentioned in the introduction, this happens for example in the case where $\mathcal{Z}$ models a thin layer of metal. Actually, the $\lambda$ part of the impedance operator has to be treated as a compact perturbation of the $\operatorname{curl}_{\Gamma} \eta \operatorname{curl}_{\Gamma}$ operator but it not true. Actually, similarly to the volume spaces, $\boldsymbol{H}^{0}\left(\operatorname{curl}_{\Gamma}, \Gamma\right)$ is not compactly embedded into $\boldsymbol{L}_{t}^{2}(\Gamma)$ since, for example, for all $p \in H^{1}(\Gamma)$ we have

$$
\operatorname{curl}_{\Gamma} \nabla_{\Gamma} p=0 .
$$

Nevertheless, we prove in what follows that $\mathcal{Z}+\boldsymbol{S}_{\Gamma}: \mathbf{V}(\Gamma) \rightarrow \mathbf{V}(\Gamma)^{*}$ is an isomorphism by using a Helmholtz' decomposition of $\mathbf{V}(\Gamma)$. Before giving the actual decomposition we need to introduce some additional notations. For any $\mathbf{f} \in \mathbf{V}(\Gamma)^{*}$, let us define the sesquilinear form $a_{\Gamma}$ on $\mathbf{V}(\Gamma) \times \mathbf{V}(\Gamma)$ and the anti-linear form $l_{\Gamma}$ on $\mathbf{V}(\Gamma)$ by

$$
\begin{gathered}
a_{\Gamma}(\mathbf{u}, \mathbf{v}):=\int_{\Gamma}\left(\eta \operatorname{curl}_{\Gamma} \mathbf{u} \operatorname{curl}_{\Gamma} \overline{\mathbf{v}}+\lambda \mathbf{u} \cdot \overline{\mathbf{v}}\right) \mathrm{d} s+\left\langle\boldsymbol{S}_{\Gamma}(\mathbf{u}), \mathbf{v}\right\rangle_{\boldsymbol{H}^{-1 / 2}\left(\operatorname{div}_{\Gamma}, \Gamma\right), \boldsymbol{H}^{-1 / 2}\left(\operatorname{curl}_{\Gamma}, \Gamma\right)} \quad \forall(\mathbf{u}, \mathbf{v}) \in(\mathbf{V}(\Gamma))^{2}, \\
l_{\Gamma}(\mathbf{v}):=\langle\mathbf{f}, \mathbf{v}\rangle_{\mathbf{V}(\Gamma)^{*}, \mathbf{V}(\Gamma)} \quad \forall \mathbf{v} \in \mathbf{V}(\Gamma) .
\end{gathered}
$$

Then, $\mathbf{u}_{\Gamma} \in \mathbf{V}(\Gamma)$ solves (3.5) if and only if

$$
a_{\Gamma}\left(\mathbf{u}_{\Gamma}, \mathbf{v}\right)=l_{\Gamma}(\mathbf{v})
$$

for all $\mathbf{v} \in \mathbf{V}(\Gamma)$.

Let us define

$$
\stackrel{\circ}{H}^{1}(\Gamma):=\left\{p \in H^{1}(\Gamma) \mid \int_{\Gamma} p \mathrm{~d} s=0\right\}
$$

the space of $H^{1}(\Gamma)$ functions with zero mean on $\Gamma$ endowed with the $H^{1}(\Gamma)$ norm and

$$
X:=\left\{\mathbf{v} \in \mathbf{V}(\Gamma) \mid \int_{\Gamma} \lambda \mathbf{v} \cdot \nabla_{\Gamma} \bar{\xi} \mathrm{d} s+\left\langle\boldsymbol{S}_{\Gamma}(\mathbf{v}), \nabla_{\Gamma} \xi\right\rangle_{\boldsymbol{H}^{-1 / 2}\left(\operatorname{div}_{\Gamma}, \Gamma\right), \boldsymbol{H}^{-1 / 2}\left(\operatorname{curl}_{\Gamma}, \Gamma\right)}=0 \quad \forall \xi \in \stackrel{\circ}{H}^{1}(\Gamma)\right\}
$$

endowed with the $\boldsymbol{H}^{0}\left(\operatorname{curl}_{\Gamma}, \Gamma\right)$ norm. These two spaces are Hilbert spaces and we denote by $\stackrel{\circ}{H}^{1}(\Gamma)^{*}$ and $X^{*}$ their dual spaces. We prove in Lemma 4.7 that

$$
\mathbf{V}(\Gamma)=\nabla_{\Gamma} \stackrel{\circ}{H}^{1}(\Gamma) \oplus X
$$


and in Lemma 4.9 that the embedding of $X$ in $\boldsymbol{L}_{t}^{2}(\Gamma)$ is compact. To this end, let us first introduce the linear and continuous operator $A_{S}: \stackrel{\circ}{H}^{1}(\Gamma) \rightarrow \stackrel{\circ}{H}^{1}(\Gamma)^{*}$ defined by

$$
\begin{aligned}
\left\langle A_{S} p, \xi\right\rangle_{H^{1}(\Gamma)^{*}, \stackrel{H}{H}^{1}(\Gamma)} & :=a_{\Gamma}\left(\nabla_{\Gamma} p, \nabla_{\Gamma} \xi\right) \\
& =\int_{\Gamma} \lambda \nabla_{\Gamma} p \cdot \nabla_{\Gamma} \bar{\xi} \mathrm{d} s+\left\langle\boldsymbol{S}_{\Gamma}\left(\nabla_{\Gamma} p\right), \nabla_{\Gamma} \xi\right\rangle_{\boldsymbol{H}^{-1 / 2}\left(\operatorname{div}_{\Gamma}, \Gamma\right), \boldsymbol{H}^{-1 / 2}\left(\operatorname{curl}_{\Gamma}, \Gamma\right)}
\end{aligned}
$$

for all $(p, \xi) \in\left(\stackrel{\circ}{H}^{1}(\Gamma)\right)^{2}$. According to the next lemma, $A_{S}$ is an isomorphism.

Lemma 4.6. If $\lambda$ satisfies Hypothesis 4.4 then $A_{S}: \stackrel{\circ}{H}^{1}(\Gamma) \rightarrow \stackrel{\circ}{H}^{1}(\Gamma)^{*}$ is an isomorphism.

Proof. Let $\lambda \in L^{\infty}(\Gamma)$ be such that Hypothesis 4.4 is satisfied. Let $C_{S}$ and $K_{S}$ be the two bounded operators from $\stackrel{\circ}{H}^{1}(\Gamma)$ into $\stackrel{\circ}{H}^{1}(\Gamma)^{*}$ defined by

$$
\begin{gathered}
\left\langle C_{S} p, \xi\right\rangle_{\stackrel{\circ}{H}^{1}(\Gamma)^{*}, \stackrel{\circ}{H}^{1}(\Gamma)}=\int_{\Gamma} \lambda\left(\nabla_{\Gamma} p \cdot \nabla_{\Gamma} \bar{\xi}+p \bar{\xi}\right) \mathrm{d} s \quad \forall(p, \xi) \in\left(\stackrel{\circ}{H}^{1}(\Gamma)\right)^{2}, \\
\left\langle K_{S} p, \xi\right\rangle_{\stackrel{\circ}{H}^{1}(\Gamma)^{*}, \stackrel{\circ}{H}^{1}(\Gamma)}=-\int_{\Gamma} \lambda p \bar{\xi} \mathrm{d} s+\left\langle\boldsymbol{S}_{\Gamma}\left(\nabla_{\Gamma} p\right), \nabla_{\Gamma} \xi\right\rangle_{\boldsymbol{H}^{-1 / 2}\left(\operatorname{div}_{\Gamma}, \Gamma\right), \boldsymbol{H}^{-1 / 2}\left(\operatorname{curl}_{\Gamma}, \Gamma\right)} \quad \forall(p, \xi) \in\left(\stackrel{\circ}{H}^{1}(\Gamma)\right)^{2},
\end{gathered}
$$

then $A_{S}=C_{S}+K_{S}$. First of all, from (4.1) and since the imaginary part of $\lambda$ does not change sign, we have for all $p \in \stackrel{\circ}{H}^{1}(\Gamma)$

$$
\left|\left\langle C_{S} p, p\right\rangle_{H^{1}(\Gamma)^{*}, H^{1}(\Gamma)}\right| \geq \frac{1}{\sqrt{2}} \int_{\Gamma}[-\Re(\lambda)+|\Im(\lambda)|]\left(\left|\nabla_{\Gamma} p\right|^{2}+|p|^{2}\right) \mathrm{d} s \geq \frac{c}{\sqrt{2}}\|p\|_{H^{1}(\Gamma)}
$$

where $c$ is the lower bound on the modulus of $\lambda$ and $c>0$ from Hypothesis 4.4. Hence $C_{S}$ is an isomorphism from Lax-Milgram Lemma.

We prove that $K_{S}: \stackrel{\circ}{H}^{1}(\Gamma) \rightarrow \stackrel{\circ}{H}^{1}(\Gamma)^{*}$ is compact. Let $\left(p_{n}\right)_{n}$ be a bounded sequence of $\stackrel{\circ}{H}^{1}(\Gamma)$, let us prove that we can extract from $\left(K_{S} p_{n}\right)_{n}$ a subsequence that converges in $\stackrel{H}{ }^{1}(\Gamma)^{*}$. From the definition of $K_{S}$ and using the continuity of $\boldsymbol{S}_{\Gamma}: \boldsymbol{H}^{-1 / 2}\left(\operatorname{curl}_{\Gamma}, \Gamma\right) \rightarrow \boldsymbol{H}^{-1 / 2}\left(\operatorname{div}_{\Gamma}, \Gamma\right)$ we deduce that it exists a constant $C>0$ such that for all $n \in \mathbb{N}$ and for all $\xi \in \stackrel{\circ}{H}^{1}(\Gamma)$ we have

$$
\left\langle K_{S} p_{n}, \xi\right\rangle_{\dot{H}^{1}(\Gamma)^{*}, \stackrel{\circ}{H}^{1}(\Gamma)} \leq\|\lambda\|_{L^{\infty}(\Gamma)}\left\|p_{n}\right\|_{L^{2}(\Gamma)}\|\xi\|_{L^{2}(\Gamma)}+C\left\|\nabla_{\Gamma} p_{n}\right\|_{\boldsymbol{H}^{-1 / 2}\left(\operatorname{curl}_{\Gamma}, \Gamma\right)}\left\|\nabla_{\Gamma} \xi\right\|_{\boldsymbol{H}^{-1 / 2}\left(\operatorname{curl}_{\Gamma}, \Gamma\right)} .
$$

But since $\operatorname{curl}_{\Gamma}\left(\nabla_{\Gamma} p_{n}\right)=0$ we deduce that $\left\|\nabla_{\Gamma} p_{n}\right\|_{\boldsymbol{H}^{-1 / 2}\left(\operatorname{curl}_{\Gamma}, \Gamma\right)}=\left\|\nabla_{\Gamma} p_{n}\right\|_{\boldsymbol{H}_{t}^{-1 / 2}(\Gamma)}$. Similarly, we obtain that it exists $C>0$ such that for all $n \in \mathbb{N}$ :

$$
\left\|\nabla_{\Gamma} \xi\right\|_{H^{-1 / 2}\left(\operatorname{curl}_{\Gamma}, \Gamma\right)}=\left\|\nabla_{\Gamma} \xi\right\|_{\boldsymbol{H}_{t}^{-1 / 2}(\Gamma)} \leq C\|\xi\|_{H^{1 / 2}(\Gamma)} .
$$

Therefore, we obtain that it exists $C>0$ such that for all $n \in \mathbb{N}$ :

$$
\left\|K_{S} p_{n}\right\|_{\dot{H}^{1}(\Gamma)^{*}} \leq C\left(\left\|p_{n}\right\|_{L^{2}(\Gamma)}+\left\|\nabla_{\Gamma} p_{n}\right\|_{\boldsymbol{H}_{t}^{-1 / 2}(\Gamma)}\right) \leq C\left\|p_{n}\right\|_{H^{1 / 2}(\Gamma)} .
$$

We recall that the sequence $\left(p_{n}\right)_{n}$ is bounded in $H^{1}(\Gamma)$ and therefore one can extract from $\left(p_{n}\right)_{n}$ a subsequence still denoted by $\left(p_{n}\right)_{n}$ that is of Cauchy type in $H^{1 / 2}(\Gamma)$. This observation together with inequality (4.2) implies that $K_{S}$ is compact and therefore that $A_{S}=C_{S}+K_{S}$ is of Fredholm type with index 0 since $C_{S}$ is an isomorphism.

To conclude the proof, let us prove that $A_{S}$ is injective. We take $p \in \stackrel{\circ}{H}^{1}(\Gamma)$ such that $A_{S} p=0$. We then have

$$
\int_{\Gamma} \lambda\left|\nabla_{\Gamma} p\right|^{2} \mathrm{~d} s+\left\langle\boldsymbol{S}_{\Gamma}\left(\nabla_{\Gamma} p\right), \nabla_{\Gamma} p\right\rangle_{\boldsymbol{H}^{-1 / 2}\left(\operatorname{div}_{\Gamma}, \Gamma\right), \boldsymbol{H}^{-1 / 2}\left(\operatorname{curl}_{\Gamma}, \Gamma\right)}=0 .
$$


THE ELECTROMAGNETIC SCATTERING PROBLEM WITH GENERALIZED IMPEDANCE BOUNDARY CONDITIONS 915

Let $(\mathbf{E}, \mathbf{H})$ be the unique solution to (3.4) with $\mathbf{v}=\nabla_{\Gamma} p$ on $\Gamma$. Integration by part formula (2.4) implies that

$$
\begin{aligned}
\Re\left\langle\boldsymbol{\nu} \times \mathbf{E}, \mathbf{H}_{T}\right\rangle_{\boldsymbol{H}^{-1 / 2}\left(\operatorname{div}_{\Gamma}, \Gamma\right), \boldsymbol{H}^{-1 / 2}\left(\operatorname{curl}_{\Gamma}, \Gamma\right)=} & -\Re\left(\int_{\partial B_{R}} \hat{x} \times \mathbf{E} \cdot \overline{\mathbf{H}} \mathrm{d} s\right) \\
& +\int_{\Omega_{\mathrm{ext}} \cap B_{R}} \Re(i \kappa \mu)|\mathbf{H}|^{2}+\Re(i \kappa \epsilon)|\mathbf{E}|^{2} \mathrm{~d} s
\end{aligned}
$$

for every ball $B_{R}$ that is such that $\bar{\Omega} \subset B_{R}$. Since $\mathbf{H}_{T}=\nabla_{\Gamma} p$ and $\boldsymbol{\nu} \times \mathbf{E}=\boldsymbol{S}_{\Gamma}\left(\mathbf{H}_{T}\right)$ on $\Gamma$, this last equality becomes

$$
\begin{aligned}
\Re\left(\int_{\partial B_{R}} \hat{x} \times \mathbf{E} \cdot \overline{\mathbf{H}} \mathrm{d} s\right)= & -\Re\left\langle\boldsymbol{S}_{\Gamma}\left(\nabla_{\Gamma} p\right), \nabla_{\Gamma} p\right\rangle_{\boldsymbol{H}^{-1 / 2}\left(\operatorname{div}_{\Gamma}, \Gamma\right), \boldsymbol{H}^{-1 / 2}\left(\operatorname{curl}_{\Gamma}, \Gamma\right)} \\
& +\int_{\Omega_{\mathrm{ext}} \cap B_{R}} \Re(i \kappa \mu)|\mathbf{H}|^{2}+\Re(i \kappa \epsilon)|\mathbf{E}|^{2} \mathrm{~d} s .
\end{aligned}
$$

Therefore, by using (4.3) we obtain

$$
\Re\left(\int_{\partial B_{R}} \hat{x} \times \mathbf{E} \cdot \overline{\mathbf{H}} \mathrm{d} s\right)=\int_{\Gamma} \Re(\lambda)\left|\nabla_{\Gamma} p\right|^{2} \mathrm{~d} s+\int_{\Omega_{\mathrm{ext}} \cap B_{R}} \Re(i \kappa \mu)|\mathbf{H}|^{2}+\Re(i \kappa \epsilon)|\mathbf{E}|^{2} \mathrm{~d} s .
$$

Since $\Re(\lambda) \leq 0, \Re(i \kappa \mu) \leq 0$ and $\Re(i \kappa \epsilon) \leq 0$ we have

$$
\Re\left(\int_{\partial B_{R}} \hat{x} \times \mathbf{E} \cdot \overline{\mathbf{H}} \mathrm{d} s\right) \leq 0
$$

and Rellich's lemma together with the unique continuation principle (see [17]) implies that $\mathbf{E}=\mathbf{H}=0$ in $\Omega_{\mathrm{ext}}$ and as a consequence $\nabla_{\Gamma} p=0$. Since $p$ has a zero mean on $\Gamma$, this implies $p=0$ which concludes the proof.

We make use of the isomorphism $A_{S}$ to prove the following Helmholtz' decomposition that has been obtained in [10] for regular boundaries and functions $\lambda$.

Lemma 4.7. If $\lambda$ satisfies Hypothesis 4.4 then $\boldsymbol{V}(\Gamma)$ writes as the direct sum of $\nabla_{\Gamma} \stackrel{\circ}{H}^{1}(\Gamma)$ and $X$ :

$$
\boldsymbol{V}(\Gamma)=\nabla_{\Gamma} \stackrel{\circ}{H}^{1}(\Gamma) \oplus X
$$

and there exists $C>0$ such that

$$
\|\boldsymbol{w}\|_{\boldsymbol{V}(\Gamma)}+\left\|\nabla_{\Gamma} p\right\|_{\boldsymbol{V}(\Gamma)} \leq C\left\|\nabla_{\Gamma} p+\boldsymbol{w}\right\|_{\boldsymbol{V}(\Gamma)}
$$

for all $\boldsymbol{w} \in X$ and $p \in \stackrel{\circ}{H}^{1}(\Gamma)$.

Proof. Let us take $\mathbf{u} \in \mathbf{V}(\Gamma)$, and let us define $F$ as being the unique element of $\stackrel{\circ}{H}^{1}(\Gamma)^{*}$ that satisfies

$$
\langle F, \xi\rangle_{\mathscr{H}^{1}(\Gamma)^{*}, \stackrel{H}{H}^{1}(\Gamma)}=\int_{\Gamma} \lambda \mathbf{u} \cdot \nabla_{\Gamma} \bar{\xi} \mathrm{d} s+\left\langle\boldsymbol{S}_{\Gamma}(\mathbf{u}), \nabla_{\Gamma} \xi\right\rangle_{\boldsymbol{H}^{-1 / 2}\left(\operatorname{div}_{\Gamma}, \Gamma\right), \boldsymbol{H}^{-1 / 2}\left(\operatorname{curl}_{\Gamma}, \Gamma\right)} \quad \forall \xi \in \stackrel{\circ}{H}^{1}(\Gamma) .
$$

Since $A_{S}: \stackrel{\circ}{H}^{1}(\Gamma) \rightarrow \stackrel{\circ}{H}^{1}(\Gamma)^{*}$ is an isomorphism (Lem. 4.6), it exists a unique $p \in \stackrel{\circ}{H}^{1}(\Gamma)$ such that $A_{S} p=F$ and it exists $C>0$ such that

$$
\|p\|_{H^{1}(\Gamma)} \leq C\|\mathbf{u}\|_{\mathbf{V}(\Gamma)} .
$$

Let us define $\mathbf{w}:=\mathbf{u}-\nabla_{\Gamma} p$, from the definition of $A_{S}$ and $p$, we have

$$
\int_{\Gamma} \lambda \mathbf{w} \cdot \nabla_{\Gamma} \bar{\xi} \mathrm{d} s+\left\langle\boldsymbol{S}_{\Gamma}(\mathbf{w}), \nabla_{\Gamma} \xi\right\rangle_{\boldsymbol{H}^{-1 / 2}\left(\operatorname{div}_{\Gamma}, \Gamma\right), \boldsymbol{H}^{-1 / 2}\left(\operatorname{curl}_{\Gamma}, \Gamma\right)}=\left\langle F-A_{S} p, \xi\right\rangle_{\dot{H}^{1}(\Gamma)^{*}, \stackrel{\circ}{H}^{1}(\Gamma)}=0 \quad \forall \xi \in \stackrel{\circ}{H}^{1}(\Gamma)
$$


whence, $\mathbf{w} \in X$ and by (4.4) we have the following continuity relation

$$
\|\mathbf{w}\|_{\mathbf{V}(\Gamma)}+\left\|\nabla_{\Gamma} p\right\|_{\mathbf{V}(\Gamma)} \leq\|\mathbf{u}\|_{\mathbf{V}(\Gamma)}+2\left\|\nabla_{\Gamma} p\right\|_{\mathbf{V}(\Gamma)} \leq(2 C+1)\|\mathbf{u}\|_{\mathbf{V}(\Gamma)}
$$

for $C>0$. We have then proven that for any $\mathbf{u} \in \mathbf{V}(\Gamma)$ it exists $p \in \stackrel{\circ}{H}^{1}(\Gamma)$ and $\mathbf{w} \in X$ such that $\mathbf{u}=\nabla_{\Gamma} p+\mathbf{w}$. We now only have to prove that the sum between $\stackrel{\circ}{H}^{1}(\Gamma)$ and $X$ is direct. For $\mathbf{u}=\nabla_{\Gamma} p \in X \cap \nabla_{\Gamma} \stackrel{\circ}{H}^{1}(\Gamma)$ we have $A_{S} p=0$ since $\mathbf{u} \in X$. Hence $p=\mathbf{u}=0$ since $A_{S}$ is injective. This concludes the proof.

We now prove the compact embedding of $X$ into $\boldsymbol{L}_{t}^{2}(\Gamma)$.

Lemma 4.8. Let $\sigma$ be a $L^{\infty}(\Gamma)$ function such that $|\sigma(x)|>c>0$ for almost all $x \in \Gamma$ and that is such that its real and imaginary parts do not change sign. Then, the space $\mathbf{H}_{t, \sigma}^{1}(\Gamma):=\left\{\boldsymbol{u} \in \boldsymbol{V}(\Gamma) \mid \operatorname{div}_{\Gamma}(\sigma \boldsymbol{u}) \in L^{2}(\Gamma)\right\}$ is compactly embedded into $\mathbf{L}_{t}^{2}(\Gamma)$.

Proof. Let $\left(\mathbf{u}_{n}\right)_{n}$ be a bounded sequence in $\boldsymbol{H}_{t, \sigma}^{1}(\Gamma)$, then there exists $C>0$ such that for all $n \in \mathbb{N}$ we have

$$
\left\|\mathbf{u}_{n}\right\|_{L_{t}^{2}(\Gamma)} \leq C, \quad\left\|\operatorname{div}_{\Gamma}\left(\sigma \mathbf{u}_{n}\right)\right\|_{L^{2}(\Gamma)} \leq C \quad \text { and } \quad\left\|\operatorname{curl}_{\Gamma}\left(\mathbf{u}_{n}\right)\right\|_{L^{2}(\Gamma)} \leq C .
$$

We define $\varphi_{n}$ as being the unique function in $\stackrel{\circ}{H}^{1}(\Gamma)$ that satisfies

$$
\operatorname{div}_{\Gamma}\left(\sigma \nabla_{\Gamma} \varphi_{n}\right)=\operatorname{div}_{\Gamma}\left(\sigma \mathbf{u}_{n}\right),
$$

then $\sigma\left(\mathbf{u}_{n}-\nabla_{\Gamma} \varphi_{n}\right)$ has a vanishing surface divergence and is in $\boldsymbol{L}_{t}^{2}(\Gamma)$. As a consequence, it exists $v_{n} \in \stackrel{\circ}{H}^{1}(\Gamma)$ such that $\operatorname{curl}_{\Gamma} v_{n}=\sigma\left(\mathbf{u}_{n}-\nabla_{\Gamma} \varphi_{n}\right)$ and then $\mathbf{u}_{n}=\nabla_{\Gamma} \varphi_{n}+\frac{1}{\sigma} \operatorname{curl}_{\Gamma} v_{n}$. We now prove that we can extract a subsequence from $\left(\operatorname{curl}_{\Gamma} v_{n}\right)_{n \in \mathbb{N}}$ and from $\left(\nabla_{\Gamma} \varphi_{n}\right)_{n \in \mathbb{N}}$ that converge in $\boldsymbol{L}_{t}^{2}(\Gamma)$.

First of all, since (4.5) has a unique solution in $\stackrel{H}{H}^{1}(\Gamma)$ that depends continuously on the right-hand side, it exists $C>0$ such that $\left\|\varphi_{n}\right\|_{H^{1}(\Gamma)} \leq C\left\|\operatorname{div}_{\Gamma}\left(\sigma \mathbf{u}_{n}\right)\right\|_{L^{2}(\Gamma)}$. The sequence $\left(\varphi_{n}\right)_{n \in \mathbb{N}}$ is in particular bounded in $H^{1}(\Gamma)$ therefore we can extract from it a subsequence still denoted by $\left(\varphi_{n}\right)_{n \in \mathbb{N}}$ that converges in $L^{2}(\Gamma)$. We prove next that it is of Cauchy type in $H^{1}(\Gamma)$. Let us define $\varphi_{n m}:=\varphi_{n}-\varphi_{m}$ and $f_{n m}:=\operatorname{div}_{\Gamma}\left(\sigma \mathbf{u}_{n}\right)-\operatorname{div}_{\Gamma}\left(\sigma \mathbf{u}_{m}\right)$, then there exists $C>0$ such that

$$
\left\|\nabla_{\Gamma} \varphi_{n m}\right\|_{L_{t}^{2}(\Gamma)}^{2} \leq C\left|\int_{\Gamma} \sigma \nabla_{\Gamma} \varphi_{n m} \cdot \nabla_{\Gamma} \overline{\varphi_{n m}} \mathrm{~d} s\right|=C\left|\int_{\Gamma} f_{n m} \overline{\varphi_{n m}} \mathrm{~d} s\right| .
$$

Since $f_{n m}$ is bounded in $L^{2}(\Gamma)$ and $\left(\varphi_{n}\right)_{n \in \mathbb{N}}$ is a Cauchy sequence in $L^{2}(\Gamma)$, we obtain that $\left(\nabla_{\Gamma} \varphi_{n}\right)_{n \in \mathbb{N}}$ is a Cauchy sequence in $\boldsymbol{L}_{t}^{2}(\Gamma)$ whence $\left(\varphi_{n}\right)_{n \in \mathbb{N}}$ converges in $H^{1}(\Gamma)$.

Concerning $\left(v_{n}\right)_{n \in \mathbb{N}}$ we proceed in a similar way. First of all, it exists $C>0$ such that $\left\|\operatorname{curl}_{\Gamma} v_{n}\right\|_{L_{t}^{2}(\Gamma)}=$ $\left\|\nabla_{\Gamma} v_{n}\right\|_{L_{t}^{2}(\Gamma)} \geq C\left\|v_{n}\right\|_{H^{1}(\Gamma)}$ since $\Gamma$ is $C^{1,1}$ (this is still true for a Lipschitz boundary). But, we recall that $\operatorname{curl}_{\Gamma} v_{n}=\sigma\left(\mathbf{u}_{n}-\nabla_{\Gamma} \varphi_{n}\right)$, therefore it is a bounded sequence in $\boldsymbol{L}_{t}^{2}(\Gamma)$. From the compact embedding of $H^{1}(\Gamma)$ in $L^{2}(\Gamma)$, we deduce that we can extract from $\left(v_{n}\right)_{n \in \mathbb{N}}$ a subsequence still denoted $\left(v_{n}\right)_{n \in \mathbb{N}}$ that converges in $L^{2}(\Gamma)$. As previously, we conclude by proving that $\left(\operatorname{curl}_{\Gamma} v_{n}\right)_{n \in \mathbb{N}}$ is a Cauchy sequence in $\boldsymbol{L}_{t}^{2}(\Gamma)$. Let us denote $f_{n m}:=\operatorname{curl}_{\Gamma}\left(\mathbf{u}_{n}\right)-\operatorname{curl}_{\Gamma}\left(\mathbf{u}_{m}\right)$, there exists $C>0$ such that

$$
\left\|\operatorname{curl}_{\Gamma} v_{n m}\right\|_{\left(\boldsymbol{L}_{t}^{2}(\Gamma)\right)^{3}}^{2} \leq C\left|\int_{\Gamma} \sigma^{-1} \operatorname{curl}_{\Gamma} v_{n m} \cdot \operatorname{curl}_{\Gamma} \overline{v_{n m}} \mathrm{~d} s\right|=C\left|\int_{\Gamma} f_{n m} \overline{v_{n m}}, \mathrm{~d} s\right|
$$

whence $\operatorname{curl}_{\Gamma} v_{n}$ is a Cauchy sequence in $\boldsymbol{L}_{t}^{2}(\Gamma)$ and it converges in $\boldsymbol{L}_{t}^{2}(\Gamma)$. This concludes the proof since we have proven that one can extract a sequence of $\left(\mathbf{u}_{n}\right)_{n \in \mathbb{N}}$ that converges in $\boldsymbol{L}_{t}^{2}(\Gamma)$.

The following lemma definitely justifies the use of the Helmholtz' decomposition introduced in Lemma 4.7.

Lemma 4.9. If $\lambda \in L^{\infty}(\Gamma)$ satisfies Hypothesis 4.4 then the embedding of $X$ into $\mathbf{L}_{t}^{2}(\Gamma)$ is compact. 
Proof. Let $\left(\mathbf{u}_{n}\right)_{n}$ be a bounded sequence of $X$, then it exists $C>0$ such that for all $n \in \mathbb{N}$

$$
\left\|\mathbf{u}_{n}\right\|_{\mathbf{v}(\Gamma)} \leq C
$$

and since $\mathbf{u}_{n} \in X$, we also have

$$
\operatorname{div}_{\Gamma}\left(\lambda \mathbf{u}_{n}\right)=-\operatorname{div}_{\Gamma}\left(\boldsymbol{S}_{\Gamma}\left(\mathbf{u}_{n}\right)\right)
$$

in the sense of distributions. We define $\left(\mathbf{E}_{n}, \mathbf{H}_{n}\right)$ as being the unique solution to (3.4) with $\mathbf{v}=\mathbf{u}_{n}$ on $\Gamma$. By using (2.3) we have that

$$
\operatorname{div}_{\Gamma}\left(\lambda \mathbf{u}_{n}\right)=-\operatorname{div}_{\Gamma}\left(\boldsymbol{\nu} \times \mathbf{E}_{n}\right)=\boldsymbol{\nu} \cdot \operatorname{curl}_{n}=i \kappa \mu \boldsymbol{\nu} \cdot \mathbf{H}_{n} .
$$

Whence, since $\mathbf{H}_{n, T}=\mathbf{u}_{n}$, it exists $C>0$ such that

$$
\left\|\mathbf{H}_{n}\right\|_{\boldsymbol{H}(\operatorname{curl}, \Omega)} \leq C \quad \text { and } \quad\left\|\mathbf{H}_{n, T}\right\|_{L_{t}^{2}(\Gamma)} \leq C
$$

and since $\operatorname{div}\left(\mu \mathbf{H}_{n}\right)=0$ in $\Omega_{\text {ext }}$ and $\mu$ is in $C^{1}\left(\mathbb{R}^{3} \backslash \Omega\right)$, we also have

$$
\left\|\mathbf{H}_{n}\right\|_{\boldsymbol{H}(\operatorname{div}, \Omega)} \leq C \text {. }
$$

From [9] we deduce that it exists $C>0$ such that for all $n \in \mathbb{N}$

$$
\left\|\operatorname{div}_{\Gamma}\left(\lambda \mathbf{u}_{n}\right)\right\|_{L^{2}(\Gamma)}=\kappa\left\|\mu \nu \cdot \mathbf{H}_{n}\right\|_{L^{2}(\Gamma)} \leq C .
$$

Lemma 4.8 proves then that we can extract a sequence of $\left(\mathbf{u}_{n}\right)_{n}$ that converges in $\boldsymbol{L}_{t}^{2}(\Gamma)$ which finishes the proof.

We now conclude with the proof of Theorem 4.5.

Proof of Theorem 4.5. We take $\mathbf{f} \in \mathbf{V}(\Gamma)^{*}$ and $(\lambda, \eta) \in\left(L^{\infty}(\Gamma)\right)^{2}$ such that Hypothesis 4.4 is satisfied. Since $\mathbf{V}(\Gamma)=\boldsymbol{H}^{0}\left(\operatorname{curl}_{\Gamma}, \Gamma\right) \subset \boldsymbol{H}^{-1 / 2}\left(\operatorname{curl}_{\Gamma}, \Gamma\right)$, we know from Lemma 3.2 that problem (3.1) is equivalent to problem (3.5). As a consequence, it is sufficient to prove that (3.5) is well-posed. Theorem 3.4, gives uniqueness, we only have to prove existence. We look for a solution $\mathbf{u}$ that writes $\mathbf{u}=\mathbf{u}_{0}+\nabla_{\Gamma} p$ with $\mathbf{u}_{0} \in X$ and $p \in \stackrel{\circ}{H}^{1}(\Gamma)$. The function $\mathbf{u}$ has to satisfiy

$$
a_{\Gamma}(\mathbf{u}, \mathbf{v})=l_{\Gamma}(\mathbf{v}) \quad \text { for all } \mathbf{v} \in \mathbf{V}(\Gamma)
$$

which if we use test functions being gradients of functions of $H^{1}(\Gamma)$ implies that $p$ has to satisfy

$$
\left\langle A_{S} p, \xi\right\rangle_{\left.\stackrel{\circ}{1}^{1}(\Gamma)^{*}, \stackrel{\circ}{H}^{1}(\Omega)\right\rangle}=l_{\Gamma}\left(\nabla_{\Gamma} \xi\right) \quad \text { for all } \xi \in \stackrel{\circ}{H}^{1}(\Gamma) .
$$

Let us recall that $A_{S}$ is an isomorphism between $\stackrel{\circ}{H}^{1}(\Gamma)$ and $\stackrel{\circ}{H}^{1}(\Gamma)^{*}$, therefore (4.6) has a unique solution $p \in \stackrel{\circ}{H}^{1}(\Gamma)$. If now we use test functions in $X$, we obtain that $\mathbf{u}_{0}$ has to satisfy

$$
a_{\Gamma}\left(\mathbf{u}_{0}, \mathbf{v}_{0}\right)=l_{\Gamma}\left(\mathbf{v}_{0}\right)-a_{\Gamma}\left(\nabla_{\Gamma} p, \mathbf{v}_{0}\right) \quad \text { for all } \mathbf{v}_{0} \in X .
$$

Let us prove that (4.7) has a unique solution in the Hilbert space $X$. We define $C_{X}: X \rightarrow X^{*}$ and $K_{X}: X \rightarrow X^{*}$ the bounded and linear operators that satisfy

$$
\begin{gathered}
\left\langle C_{X} \mathbf{v}, \mathbf{w}\right\rangle_{X^{*}, X}=\int_{\Gamma} \eta\left(\operatorname{curl}_{\Gamma} \mathbf{v} \operatorname{curl}_{\Gamma} \overline{\mathbf{w}}+\mathbf{v} \cdot \overline{\mathbf{w}}\right) \mathrm{d} s \\
\left\langle K_{X} \mathbf{v}, \mathbf{w}\right\rangle_{X^{*}, X}=\int_{\Gamma}(-\eta+\lambda) \mathbf{v} \overline{\mathbf{w}} \mathrm{d} s+\left\langle\boldsymbol{S}_{\Gamma}(\mathbf{v}), \mathbf{w}\right\rangle_{\boldsymbol{H}^{-1 / 2}\left(\operatorname{div}_{\Gamma}, \Gamma\right), \boldsymbol{H}^{-1 / 2}\left(\operatorname{curl}_{\Gamma}, \Gamma\right)}
\end{gathered}
$$


for all $\mathbf{v}, \mathbf{w} \in X$. With these definitions $\left\langle\left(C_{X}+K_{X}\right) \mathbf{v}, \mathbf{w}\right\rangle_{X^{*}, X}=a_{\Gamma}(\mathbf{v}, \mathbf{w})$ for all $\mathbf{v}, \mathbf{w} \in X$. From Theorem 3.4 we know that $C_{X}+K_{X}$ is injective, let us prove that $C_{X}+K_{X}$ is a Fredholm type operator of index 0 . First of all, since $\eta$ satisfies Hypothesis 4.4 and in particulars since its imaginary and real parts do not change sign on $\Gamma$, by using inequality (4.1) we have for all $\mathbf{v} \in X$

$$
\left|\left\langle C_{X} \mathbf{v}, \mathbf{v}\right\rangle_{X^{*}, X}\right| \geq \frac{1}{\sqrt{2}} \int_{\Gamma}(-\Re(\eta)+|\Im(\eta)|)\left(\left|\operatorname{curl}_{\Gamma} \mathbf{v}\right|^{2}+|\mathbf{v}|^{2}\right) \mathrm{d} s \geq c\|\mathbf{v}\|_{H^{0}\left(\operatorname{curl}_{\Gamma}, \Gamma\right)}^{2}
$$

therefore $C_{X}$ is coercive on $X$. Moreover, $\boldsymbol{S}_{\Gamma}: \mathbf{V}(\Gamma) \rightarrow \mathbf{V}(\Gamma)^{*}$ is compact and since the injection of $X$ in $\boldsymbol{L}_{t}^{2}(\Gamma)$ is compact (Lem. 4.9) we deduce that $K_{X}$ is a compact operator. This guaranties well-posedness of (4.7) which has a unique solution $\mathbf{u}_{0} \in X$ that depends continuously on $p$ and $\mathbf{f}$. To conclude, we built a function $\mathbf{u}=\mathbf{u}_{0}+\nabla_{\Gamma} p$ that solves (3.5). We obtain the continuous dependence of $\mathbf{u}$ with respect to $\mathbf{f}$ by using Lemma 4.7 together with the fact that $A_{S}: \stackrel{\circ}{H}^{1}(\Omega) \rightarrow \stackrel{\circ}{H}^{1}(\Omega)^{*}$ and $C_{X}+K_{X}: X \rightarrow X^{*}$ are isomorphisms.

\subsection{The case of $\mathcal{Z}=\nabla_{\Gamma} \gamma \operatorname{div}_{\Gamma}+\lambda$}

We conclude this serie of examples with a third one for which we cannot use the surface formulation (3.5). Let us consider

$$
\mathcal{Z}=\nabla_{\Gamma} \gamma \operatorname{div}_{\Gamma}+\lambda
$$

for $(\lambda, \gamma)$ two functions of $L^{\infty}(\Gamma)$. It is a continuous operator from $\mathbf{V}(\Gamma):=\boldsymbol{H}^{0}\left(\operatorname{curl}_{\Gamma}, \Gamma\right)$ into its dual and for $\mathbf{v}, \mathbf{w}$ in $\mathbf{V}(\Gamma)$ we have:

$$
\langle\mathcal{Z} \mathbf{v}, \mathbf{w}\rangle_{\mathbf{V}(\Gamma)^{*}, \mathbf{V}(\Gamma)}=\int_{\Gamma}-\gamma \operatorname{div}_{\Gamma} \mathbf{v} \overline{\operatorname{div}_{\Gamma} \mathbf{w}}+\lambda \mathbf{v} \cdot \overline{\mathbf{w}} \mathrm{d} s .
$$

Since $\mathbf{V}(\Gamma)$ is not included into $\boldsymbol{H}^{-1 / 2}\left(\operatorname{curl}_{\Gamma}, \Gamma\right)$ we cannot use the formulation (3.5) in this case. Nevertheless, we show that under appropriate sign assumptions on $\lambda$ and $\gamma$ we can apply Theorem 3.6.

Hypothesis 4.10. The functions $(\lambda, \gamma) \in\left(L^{\infty}(\Gamma)\right)^{2}$ are such that

$$
\Re(\lambda) \leq 0, \quad \Re(\gamma) \geq 0 \quad \text { a.e. on } \Gamma,
$$

and it exists $c>0$ such that

$$
\Im(\lambda) \leq c, \quad \Im(\gamma) \geq-c \quad \text { a.e. on } \Gamma .
$$

Under these restrictive sign assumptions (compare to the two previous examples) $\mathcal{Z}$ satisfies assumptions of Theorem 3.6 and we have the following result.

Theorem 4.11. Let $(\lambda, \gamma) \in\left(L^{\infty}(\Gamma)\right)^{2}$ be such that Hypothesis 4.10 is satisfied. Then for all $\boldsymbol{f} \in \boldsymbol{V}(\Gamma)^{*}$ problem (3.1) has a unique solution $(\boldsymbol{E}, \boldsymbol{H})$ and for every ball $B_{R}$ that contains $\bar{\Omega}$ it exists $C_{R}>0$ such that

$$
\left\|\boldsymbol{E}^{s}\right\|_{\mathbf{H}\left(\operatorname{curl}, \Omega_{R}\right)}+\left\|\boldsymbol{H}^{s}\right\|_{V_{H, R}} \leq C\|\boldsymbol{f}\|_{\boldsymbol{V}(\Gamma)^{*}} .
$$

Remark 4.12. We can generalise further the results of this section to the case of a vanishing functions $\lambda$ and $\gamma$ on $\Gamma$. In this case we use

$$
\mathbf{V}(\Gamma):=\left\{\left.\mathbf{v} \in \boldsymbol{H}^{-1 / 2}\left(\operatorname{curl}_{\Gamma}, \Gamma\right) \quad\left|\int_{\Gamma}\right| \lambda|| \mathbf{v}\right|^{2}+|\gamma|\left|\operatorname{div}_{\Gamma} \mathbf{v}\right|^{2} \mathrm{~d} s<+\infty\right\}
$$

endowed with the norm

$$
\|\mathbf{v}\|_{\mathbf{V}(\Gamma)}^{2}:=\|\mathbf{v}\|_{\boldsymbol{H}^{-1 / 2}\left(\operatorname{curl}_{\Gamma}, \Gamma\right)}^{2}+\int_{\Gamma}|\lambda \| \mathbf{v}|^{2}+|\gamma|\left|\operatorname{div}_{\Gamma} \mathbf{v}\right|^{2} \mathrm{~d} s .
$$

Existence and uniqueness is then ensured as soon as

$$
\Re(\lambda) \leq 0, \quad \Re(\gamma) \geq 0 \quad \text { a.e. on } \Gamma,
$$


and

$$
\Im(\lambda) \leq 0, \quad \Im(\gamma) \geq 0 \quad \text { a.e. on } \Gamma .
$$

The question of existence of a solution when $\lambda$ or $\gamma$ have a negative imaginary part cannot be treated in this way and to the knowledge of the author is still open.

\section{Appendix - PROOF of TheOrem 3.6}

First of all, uniqueness holds from Theorem 3.4. To prove existence we adapt the procedure presented in ([15], Chap. 10) in the case of a Dirichlet type boundary condition to the volume formulation (3.2). We do not give a precise proof but we only highlight the main steps since it is rather classical. As stated in Section 3.1, the electromagnetic field $(\mathbf{E}, \mathbf{H})$ solves (3.2) if and only if $\mathbf{H}$ solves the variational formulation (3.3). Let $R>R_{0}$ be such that the ball $B_{R}$ contains $\bar{\Omega}$. To study equation (3.3) we introduce a Helmholtz' decomposition for $\boldsymbol{H}\left(\operatorname{curl}, \Omega_{R}\right)$ in order to handle the $L^{2}\left(\Omega_{R}\right)$ contribution that is not a compact perturbation of the principal part. Let us introduce the space of functions in $H^{1}\left(\Omega_{R}\right)$ with vanishing trace on $\Gamma$

$$
H_{0, \Gamma}^{1}\left(\Omega_{R}\right):=\left\{p \in H^{1}\left(\Omega_{R}\right) \mid p=0 \text { on } \Gamma\right\}
$$

and the following Hilbert space

$$
\begin{aligned}
X_{R} & :=\left\{\mathbf{u} \in V_{\mathbf{H}, R} \mid\right. \\
& \left.\int_{\Omega_{R}} \kappa^{2} \mu \mathbf{u} \cdot \nabla \bar{\xi} \mathrm{d} x+i \kappa\left\langle\boldsymbol{S}_{R}(\hat{x} \times \mathbf{u}), \nabla_{\partial B_{R}} \xi\right\rangle_{\boldsymbol{H}^{-1 / 2}\left(\operatorname{div}_{\partial B_{R}}, \partial B_{R}\right), \boldsymbol{H}^{-1 / 2}\left(\operatorname{curl}_{\partial B_{R}}, \partial B_{R}\right)}=0 \forall \xi \in H_{0, \Gamma}^{1}\left(\Omega_{R}\right)\right\} .
\end{aligned}
$$

Let us define the operator $A_{R}: H_{0, \Gamma}^{1}\left(\Omega_{R}\right) \rightarrow H_{0, \Gamma}^{1}\left(\Omega_{R}\right)^{*}$ characterised by

$$
\begin{aligned}
& \left\langle A_{R} p, \xi\right\rangle_{H_{0, \Gamma}^{1}\left(\Omega_{R}\right)^{*}, H_{0, \Gamma}^{1}\left(\Omega_{R}\right)}:= \\
& \quad \int_{\Omega_{R}} \kappa^{2} \mu \nabla p \cdot \nabla \bar{\xi} \mathrm{d} x+i \kappa\left\langle\boldsymbol{S}_{R}\left(\hat{x} \times \nabla_{\partial B_{R}} p\right), \nabla_{\partial B_{R}} \xi\right\rangle_{\boldsymbol{H}^{-1 / 2}\left(\operatorname{div}_{\partial B_{R}}, \partial B_{R}\right), \boldsymbol{H}^{-1 / 2}\left(\operatorname{curl}_{\partial B_{R}}, \partial B_{R}\right)}
\end{aligned}
$$

for all $p, \xi$ in $H_{0, \Gamma}^{1}\left(\Omega_{R}\right)$. Due to the symmetry of Maxwell's equations, the Magnetic-to-Electric map $\boldsymbol{S}_{R}$ is equal to $-G_{R}$ where $G_{R}$ is the Electric-to-Magnetic map which maps $\mathbf{E}_{T}$ to $\hat{x} \times \mathbf{H}$ where $(\mathbf{E}, \mathbf{H})$ solves Maxwell's equations outside $B_{R}$ together with the Silver-Müller radiation condition. As a consequence, we can use the results of [15] Lemmas 9.23 and 9.24 that state that it exists $\widetilde{\boldsymbol{S}}_{R}: \boldsymbol{H}^{-1 / 2}\left(\operatorname{div}_{\partial B_{R}}, \partial B_{R}\right) \rightarrow \boldsymbol{H}^{-1 / 2}\left(\operatorname{div}_{\partial B_{R}}, \partial B_{R}\right)$ such that for all $\mathbf{u} \in \boldsymbol{H}^{-1 / 2}\left(\operatorname{div}_{\partial B_{R}}, \partial B_{R}\right)$ we have

$$
\left\langle\widetilde{\boldsymbol{S}}_{R} \mathbf{u}, \mathbf{u} \times \hat{x}\right\rangle_{\boldsymbol{H}^{-1 / 2}\left(\operatorname{div}_{\partial B_{R}}, \partial B_{R}\right), \boldsymbol{H}^{-1 / 2}\left(\operatorname{curl}_{\partial B_{R}}, \partial B_{R}\right)} \geq c\|\mathbf{u}\|_{\boldsymbol{H}^{-1 / 2}\left(\operatorname{div}_{\partial B_{R}}, \partial B_{R}\right)}^{2}
$$

for $c>0$ and $\boldsymbol{S}_{R}+i \kappa \widetilde{\boldsymbol{S}}_{R}: \boldsymbol{H}^{-1 / 2}\left(\operatorname{div}_{\partial B_{R}}, \partial B_{R}\right) \rightarrow \boldsymbol{H}^{-1 / 2}\left(\operatorname{div}_{\partial B_{R}}, \partial B_{R}\right)$ is a compact operator. We deduce that $A_{R}$ is an isomorphism and similarly to the proof of Lemma 4.7 we obtain the following Helmholtz' decomposition

$$
V_{\mathbf{H}, R}=X_{R} \oplus \nabla H_{0, \Gamma}^{1}\left(\Omega_{R}\right) .
$$

Moreover, $X_{R}$ is compactly embedded into $\left(L^{2}\left(\Omega_{R}\right)\right)^{3}$ (see the proof of [15], Lem. 10.4). We also remark that from the sign assumption on the real part of $i \kappa \mathcal{Z}$ and the real part of $\epsilon$, it exists $C>0$ such that

$$
\left.\left|\int_{\Omega_{R}} \epsilon^{-1}\right| \mathbf{c u r l} \mathbf{u}\right|^{2}+|\mathbf{u}|^{2} \mathrm{~d} x+i \kappa\left\langle\mathcal{Z} \mathbf{u}_{T}, \mathbf{u}_{T}\right\rangle \mathbf{V}(\Gamma)^{*}, \mathbf{V}(\Gamma) \mid \geq C\|\mathbf{u}\|_{V_{\mathbf{H}, R}}^{2}
$$

for all $\mathbf{u} \in V_{\mathbf{H}, R}$. Finally, let us recall the result of Lemma 10.5 in [15] that states that $S_{R}$ can be decomposed as $S_{R}=S_{1}+S_{2}$ where $S_{1}: \boldsymbol{H}^{-1 / 2}\left(\operatorname{div}_{\partial B_{R}}, \partial B_{R}\right) \rightarrow \boldsymbol{H}^{-1 / 2}\left(\operatorname{div}_{\partial B_{R}}, \partial B_{R}\right)$ has a positive imaginary part and $S_{2} \circ \gamma_{t, R}: X_{R} \rightarrow \boldsymbol{H}^{-1 / 2}\left(\operatorname{div}_{\partial B_{R}}, \partial B_{R}\right)$ is compact where $\gamma_{t, R} \mathbf{u}=\hat{x} \times\left.\mathbf{u}\right|_{\partial B_{R}}$ for all $\mathbf{u} \in V_{\mathbf{H}, R}$. 
We now have all the tools we need to conclude the proof. Let us build a solution that decomposes as $\mathbf{H}=\mathbf{H}_{0}+\nabla p$ where $p \in H_{0, \Gamma}^{1}\left(\Omega_{R}\right)$. If $\mathbf{H}$ solves (3.3) then $p$ has to solve $A_{R} p=0$ (there is no source term in $\Omega$ ) and therefore, $p=0$. As a consequence, $\mathbf{H}_{0}$ has to solve

$$
\left(C_{R}+K_{R}\right) \mathbf{H}_{0}=F
$$

where the operators $C_{R}: X_{R} \rightarrow X_{R}^{*}$ and $K_{R}: X_{R} \rightarrow X_{R}^{*}$ are defined by

$$
\begin{aligned}
&\left\langle C_{R} \mathbf{v}, \mathbf{w}\right\rangle_{X_{R}^{*}, X_{R}:=} \int_{\Omega_{R}} \epsilon^{-1} \operatorname{curl} \mathbf{v} \cdot \operatorname{curl} \overline{\mathbf{w}}+\mathbf{v} \cdot \overline{\mathbf{w}} \mathrm{d} x+i \kappa\langle\mathcal{Z} \mathbf{v}, \mathbf{w}\rangle_{\mathbf{V}(\Gamma)^{*}, \mathbf{V}(\Gamma)} \\
&-i \kappa\left\langle S_{1}(\hat{x} \times \mathbf{v}), \mathbf{w}\right\rangle_{\boldsymbol{H}^{-1 / 2}\left(\operatorname{div}_{\partial B_{R}}, \partial B_{R}\right), \boldsymbol{H}^{-1 / 2}\left(\operatorname{curl}_{\partial B_{R}}, \partial B_{R}\right)} \\
&\left\langle K_{R} \mathbf{v}, \mathbf{w}\right\rangle_{X_{R}^{*}, X_{R}}:=-\int_{\Omega_{R}}\left(\kappa^{2} \mu+1\right) \mathbf{v} \cdot \overline{\mathbf{w}} \mathrm{d} x-i \kappa\left\langle S_{2}(\hat{x} \times \mathbf{v}), \mathbf{w}\right\rangle_{\boldsymbol{H}^{-1 / 2}\left(\operatorname{div}_{\partial B_{R}}, \partial B_{R}\right), \boldsymbol{H}^{-1 / 2}\left(\operatorname{curl}_{\partial B_{R}}, \partial B_{R}\right)}
\end{aligned}
$$

for all $\mathbf{v}, \mathbf{w} \in X_{R}$ and $F$ is such that $\langle F, \mathbf{w}\rangle_{X_{R}^{*}, X_{R}}:=i \kappa\langle\mathbf{f}, \mathbf{w}\rangle_{\mathbf{V}(\Gamma)^{*}, \mathbf{V}(\Gamma)}$ for all $\mathbf{w} \in X_{R}$. From (4.8) and the properties of $S_{1}$ and $S_{2}$ we deduce that $C_{R}$ is coercive and $K_{R}$ is compact. The general uniqueness result Theorem 3.4 ensures that it exists $\mathbf{H}_{0}$ that solves (4.9) and that depends continuously on $F$. Therefore, it exists a unique $\mathbf{H}=\mathbf{H}_{0}$ that solves the variational formulation (3.3) and we obtain the desired result.

\section{REFERENCES}

[1] A. Bendali and K. Lemrabet, Asymptotic analysis of the scattering of a time-harmonic electromagnetic wave by a perfectly conducting metal coated with a thin dielectric shell. Asymptot. Anal. 57 (2008) 199-227.

[2] L. Bourgeois, N. Chaulet and H. Haddar, Stable reconstruction of generalized impedance boundary conditions. Inverse Probl. 27 (2011).

[3] L. Bourgeois, N. Chaulet and H. Haddar, On simultaneous identification of the shape and generalized impedance boundary condition in obstacle scattering. SIAM J. Sci. Comput. 34 (2012).

[4] F. Cakoni and R. Kress, Integral equation methods for the inverse obstacle problem with generalized impedance boundary condition. Inverse Probl. 29 (2013) 015005.

[5] M. Cessenat, Mathematical Methods in Electromagnetism: Linear Theory and Applications. World scientific publishing compagny (1996).

[6] M. Chamaillard, N. Chaulet and H. Haddar, Analysis of the factorization method for a general class of boundary conditions. J. Inverse Ill-Posed Probl. 22 (2014) 643-670.

[7] S. Chun, H. Haddar and J.S. Hesthaven, High-order accurate thin layer approximations for time-domain electromagnetics, PartII: Transmission layers. J. Comput. Appl. Math. 234 (2010) 25787-2608.

[8] D. Colton and R. Kress, Inverse acoustic and electromagnetic scattering theory. In vol. 93 of Appl. Math. Sci., 3rd edition. Springer-Verlag (1998).

[9] M. Costabel. A remark on the regularity of solutions of Maxwell's equations on Lipschitz domains. Math. Methods Appl. Sci. (1990) 365-368.

[10] B. Delourme, H. Haddar and P. Joly, On the well-posedness, stability and accuracy of an asymptotic model for thin periodic interfaces in electromagnetic scattering problems. Math. Models Methods Appl. Sci. 23 (2013) 2433-2646.

[11] M. Duruflé, H. Haddar and P. Joly, Higher order generalized impedance boundary conditions in electromagnetic scattering problems. C.R. Phys. 7 (2006) 533-542.

[12] M. Duruflé, V. Péron and C. Poignard, Thin layer models for electromagnetism. Commun. Comput. Phys. 16 (2014) $213-238$.

[13] H. Haddar and P. Joly, Stability of thin layer approximation of electromagnetic waves scattering by linear and nonlinear coatings. J. Comput. Appl. Math. 143 (2002) 201-236.

[14] H. Haddar, P. Joly and H.-M. Nguyen, Generalized impedance boundary conditions for scattering problems from strongly absorbong obstacles: the case of Maxwell's equations. Math. Models Methods Appl. Sci. 18 (2008) 1787-1827.

[15] P. Monk, Finite Elements Methods for Maxwell's Equations. Calderon Press, Oxford (2003).

[16] L. Vernhet, Boundary element solution of a scattering problem involving a generalized impedance boundary condition. Math. Methods Appl. Sci. 22 (1999) 587-603.

[17] V. Vogelsang, On the strong unique continuation principle for inequalities of maxwell type. Math. Ann. 289 (1991) $285-295$. 